\title{
Large-Scale Structure Formation: from the first non-linear objects to massive galaxy clusters
}

\author{
S. Planelles · D.R.G. Schleicher · A.M. Bykov
}

Received: ; Accepted:

\begin{abstract}
The large-scale structure of the Universe formed from initially small perturbations in the cosmic density field, leading to galaxy clusters with up to $10^{15} \mathrm{M}_{\odot}$ at the present day. Here, we review the formation of structures in the Universe, considering the first primordial galaxies and the most massive galaxy clusters as extreme cases of structure formation where fundamental processes such as gravity, turbulence, cooling and feedback are particularly relevant. The first non-linear objects in the Universe formed in dark matter halos with $10^{5}-10^{8} \mathrm{M}_{\odot}$ at redshifts $10-30$, leading to the first stars and massive black holes. At later stages, larger scales became non-linear, leading to the formation of galaxy clusters, the most massive objects in the Universe. We describe here their formation via gravitational processes, including the self-similar scaling relations, as well as the observed deviations from such self-similarity and the related non-gravitational physics (cooling, stellar feedback, AGN). While on intermediate cluster scales the self-similar model is in good agreement with the observations, deviations from such self-similarity are apparent in the core regions, where numerical simulations do not reproduce the current observational results. The latter indicates that the interaction of different feedback processes may not be correctly accounted for in current simulations. Both in the most massive clusters of galaxies as well as during the formation of the first objects in the Universe, turbulent structures and shock waves appear to be common, suggesting them to be ubiquitous in the non-linear regime.
\end{abstract}

S. Planelles

Department of Astronomy, University of Trieste, via Tiepolo 11, I-34143 Trieste, Italy

INAF - National Institute for Astrophysics, Trieste, Italy

E-mail: susana.planelles@oats.inaf.it

D.R.G. Schleicher

Institut für Astrophysik, Georg-August-Universität Göttingen, Friedrich-Hund-Platz 1, 37077 Göttingen, Germany

E-mail: dschleic@astro.physik.uni-goettingen.de

A.M. Bykov

A.F. Ioffe Institute for Physics and Technology, 194021, St.Petersburg, Russia

and St.Petersburg State Politechnical University

E-mail: byk@astro.ioffe.ru 
Keywords Cosmology: theory · early Universe - numerical simulations · galaxies: clusters · hydrodynamics $\cdot \mathrm{X}$-ray: galaxies

\section{Introduction}

The current hierarchical paradigm of structure formation is set within the spatially flat $\Lambda$ Cold Dark Matter model ( $\Lambda C D M$; Blumenthal et al. 1984) with cosmological constant, also known as the concordance model. Tight constraints on the parameters of the underlying cosmological model have now been placed thanks to the combination of different observational probes (see, e.g. Voit 2005, Allen et al. 2011, Hamilton 2013, for recent reviews). In the resulting scenario (see Planck Collaboration et al. 2013a and references therein) the Universe, whose age is estimated to be $\sim 13.8 \mathrm{Gyr}$, is composed of dark energy $\left(\Omega_{\Lambda} \approx 0.7\right)$, dark matter $\left(\Omega_{D M} \approx 0.25\right)$ and baryonic matter $\left(\Omega_{b} \approx 0.05\right)$, with a Hubble constant given by $H_{0} \approx 67 \mathrm{~km} / \mathrm{s} / \mathrm{Mpc}$. In addition, the primordial matter power spectrum seems to be characterized by a power-law index $n \approx 0.96$ with an amplitude $\sigma_{8} \approx 0.83$.

Within this paradigm, the formation of the first structures in the Universe is driven by the gravitational collapse of small inflation-induced matter density perturbations existing in the primordial matter density field. Predictions from N-body simulations (e.g. Klypin \& Shandarin 1983) have confirmed that the growth of these perturbations gives rise to the formation of a complex network of cosmic structures interconnected along walls and filaments concerning a wide range of scales.

The first structures in the Universe are expected to form at redshifts of $10-30$ in dark matter (DM) halos of $10^{5}-10^{8} \mathrm{M}_{\odot}$ (Tegmark et al. 1997, Barkana \& Loeb 2001; Glover 2005, Bromm et al. 2009). A crucial condition for these DM halos to form stars or galaxies is the ability of their gas to cool in a Hubble time. To address this question, Tegmark et al. (1997) have modeled the cooling in DM halos of different virial temperatures, showing that a virial temperature of at least $1000 \mathrm{~K}$ is required so that efficient cooling via molecular hydrogen can occur. Such a temperature corresponds to a mass scale of

$$
M_{H_{2}} \sim 10^{6.5}\left(\frac{10}{1+z}\right)^{3 / 2} M_{\odot} .
$$

Halos of this or slightly higher masses are typically referred to as the so-called minihalos, which are generally assumed to harbor the first primordial stars in the Universe. Their formation has been explored through detailed numerical simulations starting from cosmological initial conditions, following the formation of the first minihalos and their gravitational collapse, including gas chemistry and cooling, down to AU-scales or below (Abel et al. 2002; Bromm \& Larson 2004; Yoshida et al. 2008). The first such simulations typically followed only the formation of the first peak during the gravitational collapse, hinting at the formation of rather massive isolated stars of $\sim 100-300 \mathrm{M}_{\odot}$ due to the rather high accretion rates of $\sim 10^{-3} \mathrm{M}_{\odot} \mathrm{yr}^{-1}$. Subsequent studies have explored the formation of self-gravitating disks and their fragmentation at later stages (Stacy et al. 2010, Clark et al. 2011, Greif et al.|2011, 2012, Latif et al. 2013c), indicating the formation of star clusters and binaries rather than isolated stars. The resulting initial mass function (IMF) of these stars is expected to be topheavy, with characteristic masses in the range of $10-100 \mathrm{M}_{\odot}$. The studies involving sink particles further suggest that low-mass protostars can be ejected from the center of the halo via 3-body interactions, thus implying the potential presence of primordial stars with less than a solar mass that could survive until the present day. Radiative feedback seems to imply an upper mass limit of 50-100 $\mathrm{M}_{\odot}$ (Hosokawa et al. 2011; Susa 2013). 
In DM halos with virial temperatures above $10^{4} \mathrm{~K}$, an additional cooling channel is present via atomic hydrogen. In such DM halos, also referred to as atomic cooling halos, cooling is always possible via atomic hydrogen lines, helium lines or recombination cooling, while the minihalos may not be able to cool if their molecular hydrogen content is destroyed by photodissociating backgrounds (Machacek et al. 2001, Johnson et al. 2007, 2008, Schleicher et al. 2010b; Latif et al. 2011). Such halos are also more robust with respect to the first supernova explosions (Wise \& Abel 2008; Greif et al. 2010), and may thus give rise to a self-regulated mode of star formation. In the presence of a strong radiative background, for instance from a nearby galaxy, they may remain metal-free and collapse close to isothermally at $\sim 8000 \mathrm{~K}$ (Omukai 2001; Spaans \& Silk 2006; Schleicher et al. 2010b; Shang et al. 2010, Latif et al. 2011, 2013b; Prieto et al. 2013). While the initial studies followed on the collapse of the first peak (Wise et al. 2008, Regan \& Haehnelt 2009a, Shang et al. 2010), Regan \& Haehnelt (2009b) aimed at following the longer-term evolution confirming the formation of a self-gravitating disk. Latif et al. (2013a) recently pursued the first high-resolution investigation on the fragmentation of such halos on AU scales, finding that fragmentation may occur, but does not inhibit the growth of the resulting central objects. For the high accretion rates of $\sim 1 \mathrm{M}_{\odot} \mathrm{yr}^{-1}$ measured in their simulations, radiative feedback is expected to be negligible (Hosokawa et al.2012) and the formation of very massive objects with up to $10^{5} \mathrm{M}_{\odot}$ seems feasible (Schleicher et al. 2013). Such supermassive stars are expected to collapse via the post-Newtonian instability and form the progenitors of supermassive black holes (SMBHs; Shapiro \& Teukolsky 1986).

Depending on previous metal enrichment and the ambient radiation field, atomic cooling halos may also gather the proper conditions for the formation of the first galaxies. The formation and evolution of these galaxies, directly connected to the formation of the first stars and their associated radiative or supernova feedback, represent a crucial and complicated aspect of the whole cosmic history. In this sense, the main focus of this review will be on the formation of the first stars and SMBHs, and the reader is referred to the reviews by Bromm et al. (2009) and Bromm \& Yoshida (2011) concerning the formation and properties of the first galaxies.

In the hierarchical paradigm of structure formation, the first objects are the building blocks of subsequent structure formation, leading to larger galaxies and galaxy clusters through accretion and mergers (e.g. Somerville et al.2012). As a consequence of this connection, regardless of the wide range of involved scales, a number of physical processes, such as the generation of turbulence during collapse and the relevance of cooling and feedback processes, seem to be common in the formation of the different cosmic structures. Roughly speaking, the cosmic hierarchy is delimited, in terms of mass and formation time, by the first galaxies in the early Universe and the most massive galaxy clusters at the present day, whereas the bulk of galaxies generally lie in-between these extreme cases. However, a full understanding of galaxy evolution represents a complex and fundamental topic in cosmology that is being currently investigated by a considerable number of authors (see Silk \& Mamon 2012 for a recent review on the current status of galaxy formation). Given the complexity of this topic and the limited space available for this review, we avoid any description of galaxy evolution. Instead, since we are mostly interested in the role that the physics of plasma plays on the formation of cosmic structures, we will focus both on the formation of the first objects, i.e. the first stars and massive black holes, as well as on the large galaxy clusters at the present day. These extreme scenarios will allow us to illustrate the importance of cooling, turbulence and feedback during structure formation independently of the considered scales. 
Galaxy clusters are the largest nonlinear objects in the Universe today and thus a central part of the large-scale structure (LSS). Clusters of galaxies, whose total masses vary from $10^{13}$ up to $10^{15} M_{\odot}$, are characterized by very deep gravitational potential wells containing a large number of galaxies $\left(\sim 10^{2}-10^{3}\right)$ over a region of a few Mpc (see, e.g. Sarazin 1988 , for an early review on galaxy clusters). Although most of the mass in clusters is in the form of DM, a very hot and diffuse plasma, the intra-cluster medium (ICM), resides within the space between galaxies in clusters. The ICM, where the thermal plasma coexists with magnetic fields and relativistic particles, holds the major part of the baryonic matter in clusters. This cluster environment affects the evolution of the hosted galaxies by means of a number of dynamical processes such as harassment, ram-pressure stripping or galaxy mergers (e.g. see Mo et al. 2010, for a textbook on galaxy formation and evolution). The intra-cluster plasma, with typical temperatures of $T \sim 10^{7}-10^{8} \mathrm{~K}$, strongly emits X-ray radiation, causing clusters of galaxies to have high X-ray luminosities, $L_{X} \sim 10^{43}-10^{45} \mathrm{erg} / \mathrm{s}$. In addition, the ICM is quite tenuous, with electron number densities of $n_{e} \sim 10^{-4}-10^{-2} \mathrm{~cm}^{-3}$ and, although it is formed mainly of hydrogen and helium, it also holds a mean abundance of heavier elements of about $\sim 1 / 3$ of the solar abundance.

Given their typical extensions and their deep gravitational potential wells, clusters of galaxies are fundamental for our comprehension of the Universe, marking the transition between cosmological and galactic scales. Whereas on cosmological scales the growth of perturbations is mainly driven by the effects of gravity on the DM component, on galactic scales gravity operates in connection with a number of gas dynamical and astrophysical phenomena. Given such an scenario, galaxy clusters and, in particular, the hot intra-cluster plasma represent a fascinating and complex environment harboring a wide range of astrophysical and dynamical processes related to both the gravitational collapse and the baryonic physics: gravitational shock waves, gas radiative cooling, star formation (SF), gas accretion onto SMBHs hosted by massive cluster galaxies, feedback from supernovae ( $\mathrm{SNe}$ ) or active galactic nuclei (AGN), shock acceleration, magnetohydrodynamical (MHD) processes, gas turbulence, ram-pressure stripping of galaxies, thermal conduction processes, energetics associated to the populations of cosmic ray $(\mathrm{CR})$ electrons and protons, etc.

All these processes are manifested by a number of cluster observables such as the thermal X-ray emission, the Sunyaev-Zel'dovich effect (SZ; Sunyaev \& Zeldovich 1972), the spectra of galaxies, or the radio synchrotron and gamma-ray emissions associated to the population of non-thermal particles. As a consequence, galaxy clusters reside in an incomparable position within astrophysics and cosmology: while the number and distribution of clusters can be used to place constraints on the current model of cosmic structure formation, a thorough understanding of the complicated processes determining the properties of the hot intra-cluster plasma seems to be crucial to fully understand galaxy cluster observations.

In this review, we describe the formation of the large-scale structure of the Universe in the framework of the $\Lambda \mathrm{CDM}$ model. A particular focus is both on the formation of the first objects, i.e. the first stars and massive black holes, as well as on the large galaxy clusters at the present day. In both applications, we emphasize the role of gravitational as well as non-gravitational plasma physics such as turbulence, cooling, magnetic fields or feedback processes. The overall structure of this review is as follows: in $\$ 2$ we start by reviewing the basic concepts of cosmic structure formation, from the early linear evolution of small density perturbations out to the complex collapse of real overdensities; in $\$ 3$ we overview the relevance for cosmology of a proper calibration of the halo mass function; in 4 we describe the formation of the first halos in the early Universe; a brief description of the selfsimilar model of the intra-cluster plasma is done in $\$ 5$, whereas in $\$ 6$ the role played by 
non-gravitational heating and cooling processes in altering the predictions of such a model is discussed; finally, we summarize the results presented in 87

Given the limited space available for this review, we refer the reader to recent reviews about early structure formation in the Universe (e.g. Bromm \& Yoshida 2011) and cosmology with clusters of galaxies (e.g. Allen et al. 2011; Kravtsov \& Borgani 2012) for a more extensive discussion of these topics.

\section{Theory of structure formation}

In this Section we outline the main theory of cosmic structure formation through the process of gravitational instability of small initial density perturbations. We refer the reader to previous reviews or cosmology textbooks for a more detailed analysis (e.g. Peebles 1993; Coles \& Lucchin 2002, see as well Borgani 2008).

\subsection{Linear evolution of density perturbations}

The gravitational instability of a uniform and non-evolving medium versus small perturbations was first addressed by Jeans (1902). Applying this theory to an expanding Universe in the linear regime, while density perturbations are small, provides a general picture of cosmic structure formation.

Let us consider an initial density perturbation field characterized by its dimensionless density contrast:

$$
\delta(\mathbf{x})=\frac{\rho(\mathbf{x})-\bar{\rho}}{\bar{\rho}}
$$

where $\rho(\mathbf{x})$ is the matter density field at the position $\mathbf{x}$, and $\bar{\rho}=\langle\rho\rangle$ is the mean mass density of the background universe. The primordial properties of this field are determined during the inflationary epoch. In general, inflationary models predict a homogeneous and isotropic Gaussian random fluctuation field (e.g. Guth \& Pi 1982), which appears to be confirmed by observed fluctuations in the Cosmic Microwave Background (CMB; e.g. Planck Collaboration et al. 2013b).

To resolve the evolution of the initial density perturbations in an expanding Universe, the perturbed Friedmann's equations need to be solved. However, during the linear evolution the problem can be simplified. Consider that a self-gravitating and pressureless fluid dominates the matter content of an expanding Universe. In principle, these assumptions are valid if the perturbation is unstable, that is, if its scale is larger than the characteristic Jeans scale 1 , and if we deal with the evolution of DM perturbations. If the fluid is also assumed to be nonrelativistic, the Newtonian treatment can be applied. In this case, the evolution of density perturbations is described by the continuity, the Euler, and the Poisson equations:

$$
\begin{array}{r}
\frac{\partial \delta}{\partial t}+\nabla \cdot[(1+\delta) \mathbf{u}]=0 \\
\frac{\partial \mathbf{u}}{\partial t}+2 H(t) \mathbf{u}+(\mathbf{u} \cdot \nabla) \mathbf{u}=-\frac{\nabla \phi}{a^{2}} \\
\nabla^{2} \phi=4 \pi G \bar{\rho} a^{2} \delta
\end{array}
$$

\footnotetext{
1 The Jeans length, the characteristic length scale for the self-gravity of the gas, is defined as $\lambda_{J}=$ $\sqrt{\frac{15 k_{B} T}{4 \pi G \mu \rho_{\text {gas }}}}$, with $k_{B}$ the Boltzmann constant, $T$ the gas temperature, $G$ the Newton's constant, $\mu$ the mean molecular weight and $\rho_{\text {gas }}$ the mass density of the gas.
} 
where spatial derivatives are with respect to the comoving coordinate $\mathbf{x}, a(t)$ is the cosmic expansion factor such that $\mathbf{r}=a(t) \mathbf{x}$ is the proper coordinate, $\mathbf{v}=\dot{\mathbf{r}}=\dot{a} \mathbf{x}+\mathbf{u}$ is the total velocity of a fluid element (with $\dot{a} \mathbf{x}$ and $\mathbf{u}=a(t) \dot{\mathbf{x}}$ giving the Hubble flow and the peculiar velocities, respectively), $\phi(\mathbf{x})$ is the gravitational potential and $H(t)=\dot{a} / a=E(t) H_{0}$ is the time-dependent Hubble parameter. In the case of a $\Lambda \mathrm{CDM}$ cosmology, when relativistic species are neglected, $E(z)$ is given by

$$
E(z) \equiv \frac{H(t)}{H_{0}}=\left[(1+z)^{3} \Omega_{m}+(1+z)^{2}\left(1-\Omega_{m}-\Omega_{\Lambda}\right)+\Omega_{\Lambda}\right]^{1 / 2} .
$$

When small density fluctuations $(\delta<<1)$ are considered, all the non-linear terms with respect to $\delta$ and $\mathbf{u}$ can be ignored and, therefore, the above equations can be written as

$$
\frac{\partial^{2} \delta}{\partial t^{2}}+2 H(t) \frac{\partial \delta}{\partial t}=4 \pi G \bar{\rho} \delta
$$

This relation represents one of the most fundamental equations within the linear theory of gravitational collapse: it delineates the Jeans instability of a fluid with no pressure under the counter-effect of the cosmic expansion (represented by the $2 H(t) \partial \delta / \partial t$ term). Since Eq. 7 is a second order differential equation in time $t$, its solution can be written as

$$
\delta(\mathbf{x}, t)=\delta_{+}\left(\mathbf{x}, t_{i}\right) D_{+}(t)+\delta_{-}\left(\mathbf{x}, t_{i}\right) D_{-}(t),
$$

where $D_{+}(t)$ and $D_{-}(t)$ are, respectively, the growing and decaying modes of $\delta(\mathbf{x}, t)$, and $\delta_{+}\left(\mathbf{x}, t_{i}\right)$ and $\delta_{-}\left(\mathbf{x}, t_{i}\right)$ the corresponding spatial distribution of the primordial matter field. Given that the density growing modes only depend on time, the density fluctuations will evolve at the same pace throughout the cosmic volume. However, these density growing modes depend on the particular underlaying cosmology in such a way that, in different Friedmann-Lemaitre-Robertson-Walker (FLRW) universes structures will grow in a different manner.

As an example, in the case of a flat matter-dominated Einstein-de-Sitter universe (EdS, $\left.\Omega_{m}=1, \Omega_{\Lambda}=0\right)$, given that $H(t)=2 /(3 t), D_{+}(t)=\left(t / t_{i}\right)^{2 / 3} \propto a(t)$ and $D_{-}(t)=\left(t / t_{i}\right)^{-1}$. Therefore, in this particular case, cosmic expansion and gravitational instability proceed at the same rate. Contrarily, it can be shown that, in the case of a cosmological model with $\Omega_{m}<1$, such as a flat one with $\Omega_{m}=0.3$, there is an epoch, when the cosmological constant begins to be significant, at which the characteristic time-scale of expansion turns out to be shorter than in the EdS case. As a consequence, after that epoch, cosmic expansion proceeds faster than gravitational collapse, generating a minor evolution in the number of collapse objects between $z \sim 0.6$ and $z=0$ (e.g. Borgani \& Guzzo 2001). These results indicate that the observational determination of the level of evolution of collapse regions (such as galaxy clusters) provides important constraints on cosmological parameters.

We can define the two-point correlation function of $\delta(\mathbf{x})$ as $\xi(r)=\left\langle\delta\left(\mathbf{x}_{1}\right) \delta\left(\mathbf{x}_{2}\right)\right\rangle$, which depends only on the distance between the considered points, $r=\left|\mathbf{x}_{1}-\mathbf{x}_{2}\right| . \xi(r)$ describes whether the density field is more $(\xi(r)>0)$ or less $(\xi(r)<0)$ concentrated than the mean. In addition, a convenient description of $\delta(\mathbf{x})$ is given by its Fourier representation $\delta(\mathbf{k})=(2 \pi)^{-3 / 2} \int d \mathbf{x} \delta(\mathbf{x}) e^{i \mathbf{k} \cdot \mathbf{x}}$. If we also express $\xi(r)$ in Fourier space, it is easily demonstrable that its Fourier transform corresponds to the power spectrum of density fluctuations:

$$
P(k)=\left\langle|\delta(\mathbf{k})|^{2}\right\rangle=\frac{1}{2 \pi^{2}} \int d r r^{2} \xi(r) \frac{\sin k r}{k r},
$$

which does not depend on the orientation of the wave-vector $\mathbf{k}$ but on its modulus. 
$P(k)$ is a fundamental quantity that provides a full statistical description of a uniform and isotropic Gaussian field. Inflationary models predict a perturbation power spectrum of the form $P(k)=A k^{n}$, where $A$ is the normalization and $n$ the spectral index. More precisely, inflation provides a nearly Gaussian density perturbation field characterized by a scale-invariant spectrum with $n \simeq 1$ (e.g. Guth \& Pi 1982), which appears to be confirmed by measured CMB anisotropies (e.g. Planck Collaboration et al.|2013b).

A common practice in the analysis of cosmological density fields is that of using filtering functions to define spatial scales. To analyze the collapse of primordial fluctuations on scales $R \propto(M / \bar{\rho})^{1 / 3}$, giving rise to objects of mass $M$, it is useful to define a window function, $W_{R}(r)$, which filters out the modes on smaller scales, and the corresponding smoothed density field, $\delta_{R}(\mathbf{x})=\delta_{M}(\mathbf{x})=\int \delta(\mathbf{y}) W_{R}(|\mathbf{x}-\mathbf{y}|) d \mathbf{y}$. If the Fourier transform of the window function ${ }^{2}$ is $W_{R}(k)$, the variance of the perturbation field at the scale $R$ is given by

$$
\sigma_{R}^{2}=\sigma_{M}^{2}=\left\langle\delta_{R}^{2}\right\rangle=\frac{1}{2 \pi^{2}} \int d k k^{2} P(k) W_{R}^{2}(k) .
$$

In principle, whereas the functional form of $P(k)$ depends on $\Omega_{m}, \Omega_{\mathrm{b}}$, and $H_{0}$ (e.g. Eisenstein $\& \mathrm{Hu} 1999$ ), its normalization, which is related to $\sigma_{R}^{2}$, needs to be determined by observations of the cosmic LSS or of the CMB anisotropies. The most widely used parameter for this normalization is $\sigma_{8}$, which is the variance estimated within comoving spheres of radius $R=8 h^{-1} \mathrm{Mpc}$, roughly matching the typical scale of massive cluster ${ }^{3}$ As we discuss in $\$ 3$. an estimate of $\sigma_{8}$ is given by the halo mass function.

This linear approximation applies after recombination, while $\delta<<1$, to describe the evolution of density fluctuations on an initial mass scale $M \gtrsim M_{J}\left(z_{\text {rec }}\right) \sim 10^{5} M_{\odot}$. However, this linear theory can not be used to study the growth of structures in the strongly non-linear regime, where typical fluctuations reach amplitudes of about unity and overdensities with $\delta>>1$ are plausible (as an example, a cluster of galaxies corresponds to $\delta \gtrsim 100$ ). In this case, non-linear models or numerical simulations are required to solve the evolution.

\subsection{Non-linear evolution of spherical perturbations: the Spherical Top-Hat Collapse}

The only situation in which the non-linear evolution can be precisely calculated is the one addressed by the simple spherically symmetric collapse model (e.g. Gunn \& Gott 1972; Bertschinger 1985). This model resolves the evolution of a spherical density perturbation of radius $R$ into a virialized halo. Initially, the spherical perturbation is assumed to have constant overdensity and, since it is expanding with the background universe, null velocity at its border. The symmetry of this configuration allows us to treat the spherical perturbation as an isolated FLRW universe, meaning that we can describe the growth of the overdensity using the same Friedmann's equations as for cosmology.

For simplicity we consider that the background universe is described by a close EdS model, in which the perturbation radius $R(t)$ behaves in the same way as the expansion factor. Within such a model, after a short period of time the growing mode will dominate the evolution of the perturbation. At the initial time $t_{i}$, by imposing the condition of null

\footnotetext{
2 The functional form of the window function, which depends on the particular choice of filter, provides the connection between mass and smoothing scale. Two common filter functions are $W_{R}(k)=$ $3[\sin (k R)-k R \cos (k R)] /(k R)^{3}$ and $W_{R}(k)=\exp \left(-(k R)^{2} / 2\right)$ corresponding to the top-hat and the Gaussian windows, respectively. For each of these filters, the correspondent relation between mass and smoothing scale is given by $M=(4 \pi / 3) R^{3} \bar{\rho}$ and $M=\left(2 \pi R^{2}\right)^{3 / 2} \bar{\rho}$.

3 Early redshift surveys showed that $\sigma \sim 1$ for spheres of $R=8 h^{-1}$ Mpc (e.g. Davis \& Peebles 1983).
} 
velocities at the edge of the spherical region, the linear growing mode is given by $D_{+}\left(t_{i}\right)=$ $(3 / 5) \delta\left(t_{i}\right)$ and, thus, the corresponding density parameter is $\Omega_{p}\left(t_{i}\right)=\Omega\left(t_{i}\right)\left(1+\delta_{i}\right)$, where the suffix $p$ stands for the perturbation itself, and the other quantities for the unperturbed background universe.

The perturbation will grow until reaching its maximum expansion at a given turn-around time, $t_{t a}$. If at this moment the spherical perturbation detaches from the expansion of the background and, instead, initiates to collapse, the structure will be formed. By solving the Friedmann's equations it can be shown that, if $\Omega_{p}\left(t_{i}\right)>1$, the perturbation will recollapse. At $t_{t a}$, the corresponding perturbation overdensity is given by ${ }^{4} \delta_{+}\left(t_{t a}\right) \simeq 4.6$.

After $t_{t a}$, the perturbation decouples from the underlying cosmic expansion and recollapses, reaching an equilibrium state at the time $t_{v i r}$ at which the virial condition between the kinetic $K$ and the potential $U$ energy of the perturbation is satisfied, that is, $U=-2 K$. Assuming energy conservation during the evolution into this equilibrium state, the virial condition can be used to derived that $R_{t a}=2 R_{\mathrm{vir}}$ and that the density at $t_{v i r}$ is $\rho_{p}\left(t_{v i r}\right)=8 \rho_{p}\left(t_{t a}\right)$. Hence, the non-linear overdensity of the perturbation at the virialization is given by

$$
\Delta_{\text {vir }}=\frac{\rho_{p}\left(t_{\text {vir }}\right)}{\rho\left(t_{\text {vir }}\right)}=18 \pi^{2} \simeq 178 .
$$

Despite the simplicity of the spherical collapse model, this result is quite encouraging since N-body simulations find that a density contrast of $\sim 100-200$ is quite successful in defining DM halos. Indeed, in these simulations a common definition of halo mass is given by $M_{200}$, defined as the mass enclosed by a sphere with an overdensity equal to 200 .

On the contrary, the linear-theory extrapolation predicts a smaller value for the required overdensity at the time of collapse:

$$
\delta_{c}=\delta_{+}\left(t_{v i r}\right)=\delta_{+}\left(t_{t a}\right)\left(\frac{t_{v i r}}{t_{t a}}\right)^{2 / 3} \simeq 1.69 .
$$

As we will see later, this is a key discriminant that determines the halo mass function.

The above equations, valid for an EdS cosmology, can be extended to any other cosmological model. In this sense, the overdensity at virialization can be defined as $\Delta_{c}$ or as $\Delta_{v i r}$ depending on whether it is referred to the critical, $\rho_{c}(z)$, or to the mean background matter density, $\rho_{m}(z)$. These overdensities relate to each other as $\Delta_{v i r}=\Delta_{c} / \Omega_{m}(z)$ (see Bryan \& Norman 1998 for an estimation of $\Delta_{v i r}$ in open and flat $\Lambda C D M$ universes).

\subsection{Non-linear evolution of real overdensities}

Given the simplicity of the spherical collapse model, it is not adequate to properly describe the non-spherical collapse of actual overdense regions. To this end, cosmological simulations (see, e.g. Dolag et al. 2008, Borgani \& Kravtsov 2011, for details on the numerical techniques) are essential to deepen into the main properties of the real gravitational collapse.

As an example, the left panel of Fig. 1 displays the evolution of the DM density field from $z \simeq 4$ until $z=0$ as obtained from a cosmological hydrodynamical simulation. At early epochs, collapsed objects with low masses populate the proto-cluster region. As the evolution proceeds, these objects merge into larger structures at later times. As can be inferred from this figure, the actual collapse of overdense regions shows a number of complexities

\footnotetext{
${ }^{4}$ On the contrary, the linear-theory extrapolation to $t_{t a}$ yields a smaller value: $\delta_{+}\left(t_{t a}\right) \simeq 1.07$.
} 
in comparison to the top-hat collapse model: severe departures from spherical symmetry and constant density edges, filamentary matter accretion, and the existence of smaller overdensities within larger overdense already collapsing regions. Given that different overdense regions have different spatial extensions and their time evolution proceeds differently, the actual collapse of a cluster-scale overdensity is a process prolonged in time (e.g. Diemand et al. 2007). Besides, the non-linear nature of this picture gives rise to merging events and interactions between overdensities at different scales, leading to an important matter redistribution within the considered regions.

Despite the complexities associated with the process of non-linear collapse, as the evolution proceeds, the resulting collapsing regions tend to reach an equilibrium state. This state is described differently depending on the collisional or collisionless nature of the considered component. Indeed, the equilibrium state of the collisional gas component can be approximated by the condition of hydrostatic equilibrium (HE from now on), $\nabla \phi(\mathbf{x})=$ $-\nabla p(\mathbf{x}) / \rho_{\text {gas }}(\mathbf{x})$, under which pressure gradients and gravitational forces compensate each other. On the contrary, the equilibrium configuration for the collisionless dark matter component is provided by the Jeans equation (Binney \& Tremaine 2008). With the additional assumptions of spherical symmetry and an ideal gas equation of state for the ICM gas, the resulting equations (see, e.g. Kravtsov \& Borgani 2012, for a complete description) are commonly used to derive cluster masses (e.g. Ettori et al. 2013). However, a number of processes, such as continuous matter accretion or merging events, can keep clusters away from equilibrium, introducing systematic uncertainties when applying the above assumptions.

Given the complexities inherent to the non-linear process of halo formation, the definition of a DM halo is not trivial and, as a consequence, there is no single definition that is agreed upon in the literature. This incongruity has resulted in a number of different halo finding algorithms based on different halo boundaries and mass definitions (see Knebe et al. 2011: Onions et al. 2012, Knebe et al.|2013, for recent comparisons of different halo finders). In this sense, two of the most widely used halo definitions are those based on the Friendsof-Friends (FoF; Davis et al. 1985) and the Spherical Overdensity (SO; Lacey \& Cole 1994) algorithms 5

\section{The halo mass function}

The halo mass function (HMF) is the number density of collapsed objects, at redshift $z$, with mass between $M$ and $M+d M$ in a given comoving volume. While from an observational point of view the HMF is difficult to determine with high precision (e.g. Rozo et al. 2010), it can approximately be analyzed through analytic models (e.g. Press \& Schechter 1974), and it is relatively simple to study by means of N-body cosmological simulations (e.g. Cohn \& White 2008, Tinker et al. 2008, Crocce et al. 2010, Courtin et al. 2011; Bhattacharya et al. 2011; Angulo et al. 2012, Watson et al. 2013; Murray et al.|2013, for recent studies).

In this Section, after introducing the HMF as originally derived by Press \& Schechter (1974), we overview how cosmological simulations are currently used to provide more precise calibrations of this important prediction.

\footnotetext{
5 While the FoF algorithm identifies DM halos with groups of DM particles separated by a distance shorter than a given linking length parameter, the $\mathrm{SO}$ algorithm is based on the mean overdensity criterion.
} 

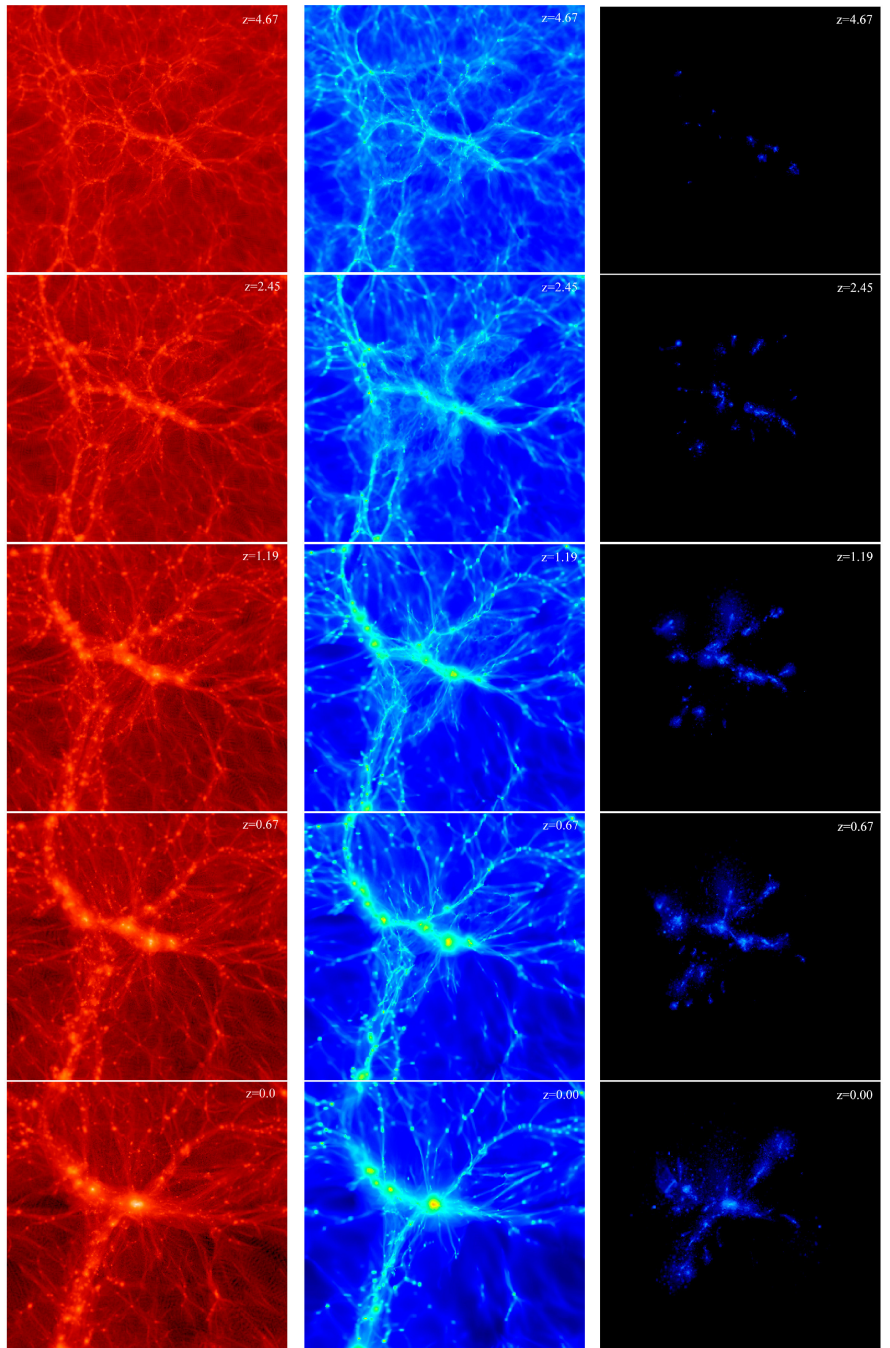

Fig. 1 Formation and evolution of galaxy clusters as described by a hydrodynamical simulation. Left, central and right columns show, respectively, the evolution of the dark matter, gas and stellar densities from $z \simeq 4$ (top panels) until $z=0$ (bottom panels). At $z=0$, the biggest cluster formed has a virial mass of $\sim 10^{15} M_{\odot}$ and a radius of $\sim 3 \mathrm{Mpc}$. The simulation was performed with the Eulerian-AMR cosmological code MASCLET Quilis 2004). Each panel is 64 comoving Mpc length per edge and 5 comoving Mpc depth.

\subsection{The Press-Schechter approach}

Press \& Schechter (1974. PS from now on), based on the spherical collapse model, performed the first analytical attempt to derive the HMF. The main idea of this formalism is that, any collapsed object with mass $\geq M$ by redshift $z$ stems from regions where $\delta_{M} \geq \delta_{c}$, being $\delta_{M}$ the linearly extrapolated density field (smoothed on a mass scale $M$ ), and $\delta_{c}$ the critical overdensity for collapse. Motivated by the spherical collapse model (see Eq. 12), 
$\delta_{c} \simeq 1.69$, being $z$-independent only in an EdS universe ${ }^{6}$. Assuming a Gaussian distribution for the initial density fluctuations, the probability of a given point to be within a region of scale $R$ satisfying the above conditions is:

$$
F(M, z)=\frac{1}{\sqrt{2 \pi} \sigma_{M}(z)} \int_{\delta_{c}}^{\infty} \exp \left(-\frac{\delta_{M}^{2}}{2 \sigma_{M}(z)^{2}}\right) d \delta_{M},
$$

where $\sigma_{M}(z)$ is the corresponding rms density fluctuation.

From the above equation, the HMF is estimated as $\partial F(M, z) / \partial M$ (the fraction of independent regions evolving into objects with mass between $M$ and $M+d M)$ divided by $M / \bar{\rho}$. An inherent implication of the PS approach is that, only overdense regions participate in the spherical collapse and, consequently, only half of the total mass content of the Universe is considered. Thus, including a missing factor of 2 , the PS mass function is given by:

$$
\begin{aligned}
\frac{d n(M, z)}{d M} & =\frac{2}{(M / \bar{\rho})} \frac{\partial F(M, z)}{\partial M}=\sqrt{\frac{2}{\pi}} \frac{\bar{\rho}}{M^{2}} \frac{\delta_{c}}{\sigma_{M}(z)}\left|\frac{d \log \sigma_{M}(z)}{d \log M}\right| \exp \left(-\frac{\delta_{c}^{2}}{2 \sigma_{M}(z)^{2}}\right)= \\
& =\frac{\bar{\rho}}{M} \psi(v),
\end{aligned}
$$

which only depends on the peak height $v \equiv \delta_{c}(z) / \sigma_{M}(z)$.

It has been shown that the functional form of $\psi(v)$ provided by the PS approach diverts significantly from the one derived from cosmological simulations (e.g. Sheth \& Tormen 1999, Jenkins et al. 2001; Tinker et al. 2010). In order to alleviate these discrepancies, a number of changes in the original model have been introduced (Bond et al. 1991; Lacey \& Cole 1993). In this regard, the HMF has been analyzed accounting for the ellipsoidal collapse (Audit et al. 1997, Sheth \& Tormen 1999, Sheth et al. 2001) or, within the excursion set theory, for non-gaussian primordial conditions (Maggiore \& Riotto 2010). However, given the relatively simple assumptions on which these analytical prescriptions rely, their accuracy to properly describe the HMF is limited.

\subsection{Using cosmological simulations to calibrate the halo mass function}

Cosmological simulations represent a powerful means to accurately calibrate the HMF (see Murray et al. 2013, for a recent comparison of different HMF available in the literature).

Given the exponential dependence of the HMF on mass and redshift, a precise calibration is extremely useful to place constraints on cosmological parameters (e.g. Allen et al. 2011, Weinberg et al. 2013 for recent reviews). As an example, whereas at low redshift the mass function of massive objects can be used to set limits on the combination of $\sigma_{8}$ and $\Omega_{\mathrm{m}}$, the redshift evolution of the HMF helps in breaking this degeneracy.

On the other hand, however, this exponential dependence makes the HMF very sensitive to the particular mass definition or to the small variations of $\delta_{c}(z)$ with redshift and cosmology. In this regard, many analysis of halo abundance by means of DM-only simulations consider that $\delta_{c}(z)$ is constant. As a consequence, they generally obtain a HMF that can be written as an almost 'universal' function of $v$, i.e., independent of redshift or cosmology (e.g. Sheth \& Tormen 1999; Jenkins et al. 2001; Evrard et al. 2002; White 2002, Warren et al. 2006, Tinker et al. 2008, Crocce et al. 2010, Bhattacharya et al. 2011; Courtin et al.

\footnotetext{
6 In the general case, however, this overdensity depends weakly on redshift and cosmology (for example, $\delta_{c} \simeq 1.675$ in a $\Lambda C D M$ model at $z=0$ ).
} 

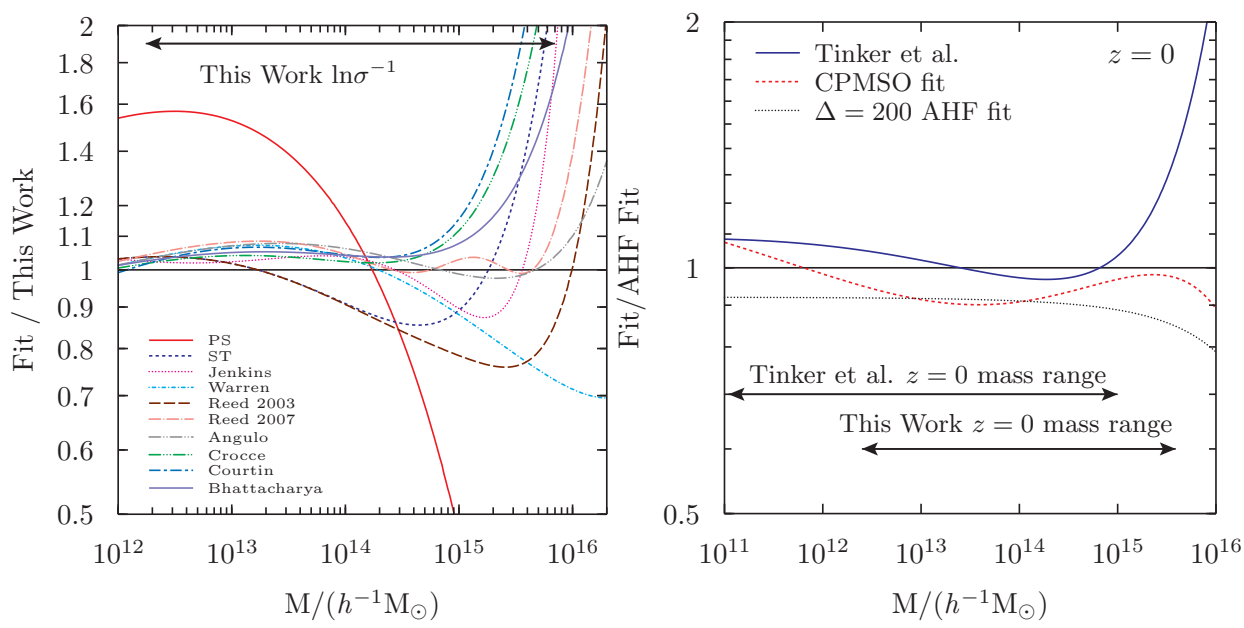

Fig. 2 Left panel: Comparison, at $z=0$, between the universal fit proposed by Watson et al. (2013) for the FoF HMF and several FoF fits available in the literature. Right panel: Comparison, at $z=0$, between the mass function obtained by Watson et al. (2013) with two different SO halo finders (AHF and CPMSO) and the fit by Tinker et al. (2008). Figures from Watson et al. (2013). In both figures, the label 'This Work' refers to the work by Watson et al. (2013).

2011). However, small deviations in $\delta_{c}(z)$ can induce important changes in the HMF and, therefore, a precise calibration of the HMF requires to account for the correct dependencies of $\delta_{c}(z)$ (e.g. Courtin et al. 2011).

In the last years, in order to reach an accurate description of the shape of the HMF, large N-body simulations have been used to evaluate the uncertainties induced by different redshifts, cosmological models and halo definitions. As an example, Watson et al. (2013), with a set of large cosmological DM-only simulations, examined the redshift evolution (out to $z=30$ ) of the HMF and its dependence on the FoF and SO halo mass definitions (see Knebe et al. 2011. for a comparison of the HMF provided by different halo finders). In this work, they showed that the SO HMF clearly evolves with redshift and obtained a $z$ parameterized fit suitable for the whole redshift interval to within $\sim 20 \%$. The right panel of Fig. 2 shows the comparison of several SO mass functions that they obtained at $z=0$. On the contrary, a weaker $z$-evolution was found for the FoF HMF. In this case, as it is shown in the left panel of Fig. 2 they obtained a 'universal' fit function ${ }^{7}$ that agrees to within $\sim 10 \%$ with a number of fits available in the literature at $z=0$, a degree of deviation in accordance with the values reported in previous works (e.g. Reed et al. 2007, Lukić et al. 2007; Tinker et al. 2008, Courtin et al. 2011,

More recently, hydrodynamical simulations have demonstrated that baryonic cooling and heating processes can also affect the HMF (e.g. Rudd et al. 2008; Stanek et al. 2009, Cui et al. 2012; Cusworth et al. 2014; Cui et al.2014). In this regard, Cui et al. (2014) recently found that, in comparison to DM-only simulations, hydrodynamical simulations accounting for cooling, star formation and SN feedback produce an increase of the HMF, while simulations including as well AGN feedback tend to reduce it by an overdensity-dependent

7 The fit obtained for the HMF based on the FoF halos takes the form: $f(\sigma)=A\left[\left(\frac{\beta}{\sigma}\right)^{\alpha}+1\right] e^{-\gamma / \sigma^{2}}$, with $A=0.282, \alpha=2.163, \beta=1.406$, and $\gamma=1.210$. This fit holds for $-0.55 \leq \ln \sigma^{-1}<1.31$, corresponding to masses within $\left[1.8 \times 10^{12}, 7.0 \times 10^{15}\right] h^{-1} \mathrm{M}_{\odot}$ at $z=0$. 
amount. This reduction is a consequence of the changes that AGN feedback induces in gas density profiles and, therefore, in halo masses. However, given our limited understanding of the physics of baryons, and in view of the large galaxy cluster surveys coming in the near future, the importance of this effect needs to be further and accurately investigated.

\section{Structure formation in the early universe}

The first objects in the Universe were expected to form in halos with $10^{5}-10^{8} \mathrm{M}_{\odot}$ at redshifts $10-30$. As discussed in the introduction, one may distinguish between the so-called minihalos, with virial temperatures of a few $\sim 1000 \mathrm{~K}$ and cooling via molecular hydrogen, and the so-called atomic cooling halos with virial temperatures of $10^{4} \mathrm{~K}$. Depending on previous metal enrichment and the ambient radiation field, the latter may form the first galaxies or directly collapse into supermassive black holes. The main focus of this review will be on the latter scenario, and the reader is referred to the reviews by Bromm et al. (2009) and Bromm \& Yoshida (2011) concerning the formation and properties of the first galaxies.

4.1 Primordial star formation in the first minihalos

A central ingredient for star formation in the first minihalos is their ability to cool via molecular hydrogen. The latter is possible since $\mathrm{H}_{2}$ may form even in primordial gas via the socalled $\mathrm{H}^{-}$channel

$$
\begin{aligned}
\mathrm{H}+e & \rightarrow \mathrm{H}^{-}+\gamma, \\
\mathrm{H}+\mathrm{H}^{-} & \rightarrow \mathrm{H}_{2}+e
\end{aligned}
$$

and the $\mathrm{H}_{2}^{+}$channel

$$
\begin{aligned}
& \mathrm{H}^{+}+H \rightarrow \mathrm{H}_{2}^{+}+\gamma, \\
& \mathrm{H}_{2}^{+}+H \rightarrow \mathrm{H}_{2}+H^{+} .
\end{aligned}
$$

In both channels, electrons need to act as catalysts in order to promote $\mathrm{H}_{2}$ formation, which is possible due to the electron freeze-out during cosmic recombination, leading to a ionization fraction of $\sim 10^{-4}$ in the primordial Universe (Peebles 1968). As shown by Saslaw \& Zipoy (1967), the latter may promote molecular hydrogen formation in molecular clouds, allowing them to cool and collapse within a Hubble time (Tegmark et al. 1997). The fact that the chemistry is out of equilibrium is thus crucial for the formation of the first structures, and detailed non-equilibrium calculations for the homogeneous Universe have been performed (e.g. Galli \& Palla 1998, Stancil et al. 1998, Puy \& Signore 2007, Schleicher et al. 2008, Coppola et al. 2012) to determine the chemical initial conditions for subsequent structure formation.

The first cosmological simulations following the formation and subsequent collapse of the first minihalos, including detailed models for the primordial chemistry and cooling, have been performed by Abel et al. (2002) and Bromm et al. (2002). These calculations were exploring the initial collapse phase where no fragmentation occurred. Yoshida et al. (2006) were the first to incorporate a more detailed treatment of the microphysics at high densities in such simulations. In particular, at number densities of $\sim 10^{8} \mathrm{~cm}^{-3}$, three-body $\mathrm{H}_{2}$ formation 


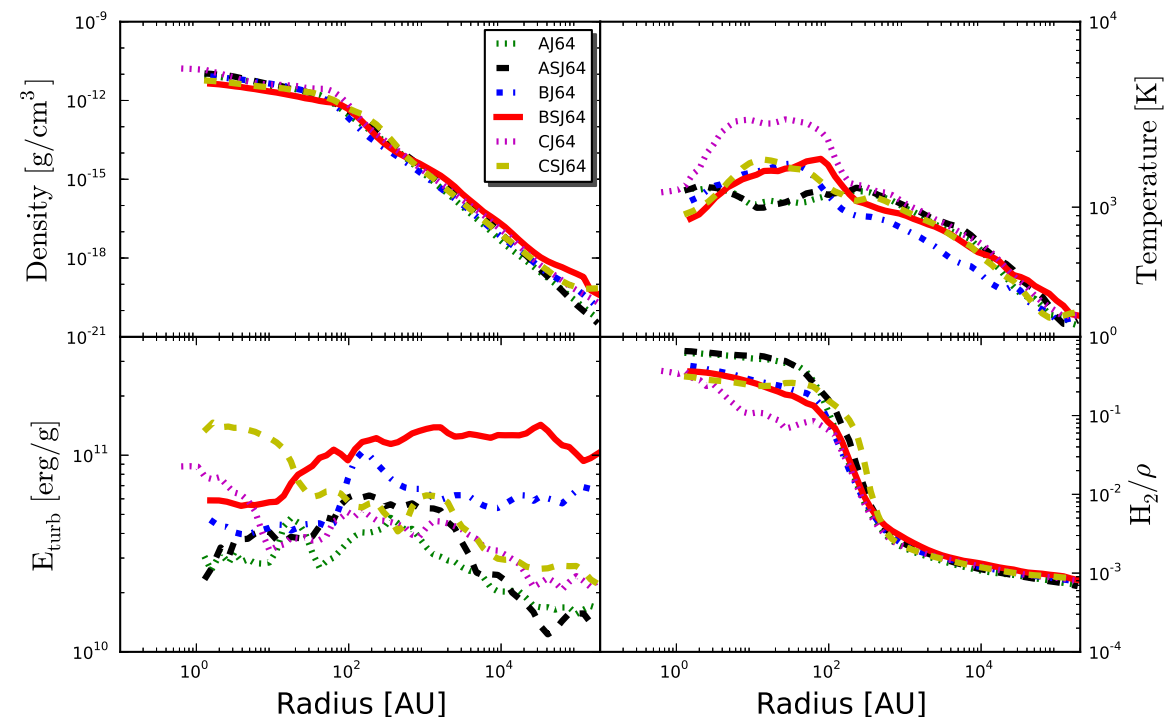

Fig. 3 Gravitational collapse in the first minihalos. Shown are radial profiles of the baryon density (upper left), gas temperature (upper right), specific turbulent energy density (lower left) and the $\mathrm{H}_{2}$ abundance (lower right). The figure shows results for three different halos A-C with and without the turbulence subgrid-scale model. Figure by Latif et al. (2013c).

rates become relevant, which turn the hydrogen gas from a predominantly atomic into a fully molecular state. The dominant three-body reactions are given as

$$
\begin{aligned}
\mathrm{H}+\mathrm{H}+\mathrm{H} & \rightarrow \mathrm{H}_{2}+H, \\
\mathrm{H}_{2}+\mathrm{H}+\mathrm{H} & \rightarrow 2 \mathrm{H}_{2} .
\end{aligned}
$$

In this review, we illustrate the collapse dynamics based on the recent simulation by Latif et al. (2013c). They employed the cosmological hydrodynamics code Enzo (O'Shea et al. 2004, Bryan et al.2014) including a detailed network for primordial chemistry (Abel et al. 1997) with updated rates and cooling functions, as well as a subgrid-scale model for hydrodynamical turbulence (Schmidt et al. 2006). Their simulation box had a total size of $300 h^{-1} \mathrm{kpc}$ with a root grid resolution of $128^{3}$, and two additional nested grids of the same resolution centered on the most massive halo. The simulation further employed 27 adaptive refinement levels, ensuring a minimum resolution of 64 cells per Jeans length. While Truelove et al. (1997) argued for a minimum resolution of 4 cells per Jeans length to avoid artificial fragmentation, more recent studies indicate a minimum resolution of 32 to 64 cells to capture the main properties of turbulence (Federrath et al. 2011; Turk et al.|2012, Latif et al. 2013b).

In Fig. 3, we show the central region of this halo when the highest refinement level is reached. The gas density follows approximately an isothermal profile with $\rho \propto r^{-2}$, and flattens on scales comparable to the Jeans length at the density peak. The temperature in- 


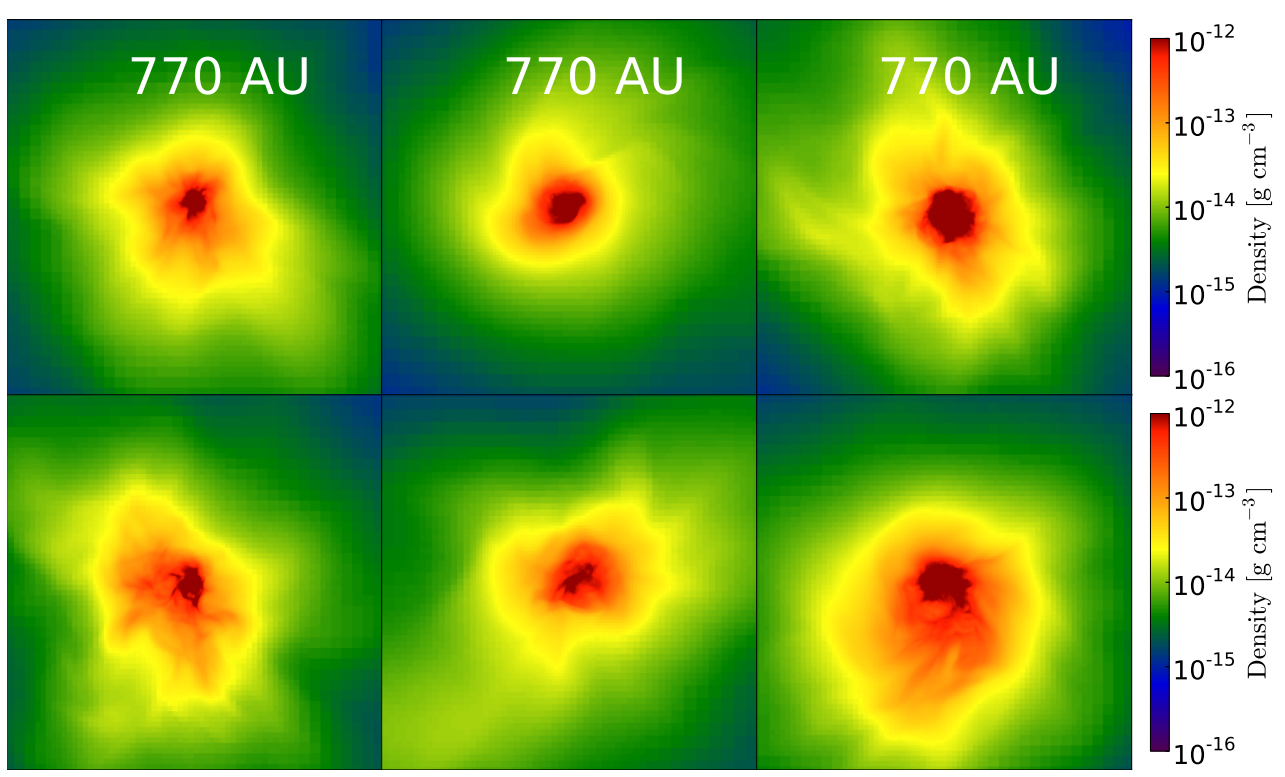

Fig. 4 Projections of the gas density in the central $770 \mathrm{AU}$ for three different halos. The top panel shows purely hydrodynamical simulations, while the simulations in the bottom panel include the subgrid-scale turbulence model by Schmidt et al. (2006). Figure by Latif et al. (2013c).

creases towards smaller radii due to gravitational compression and increasing optical depth, and reaches a temperature of $\sim 1000 \mathrm{~K}$ at densities of $\sim 10^{9} \mathrm{~cm}^{-3}$ when three-body $\mathrm{H}_{2}$ formation becomes relevant. The specific turbulent energy appears almost indepedent of scale, implying that turbulence is continuously re-generated during gravitational collapse via shocks and shear flows. The $\mathrm{H}_{2}$ abundance is of the order $10^{-3}$ on larger spatial scales, and increases rather steeply at $\sim 100 \mathrm{AU}$ when the gas becomes fully molecular as a result of the three-body reactions. The corresponding density projections in the central $770 \mathrm{AU}$ are given in Fig. 4 for three different halos in simulations with and without the turbulence subgrid-scale model. At this stage of the simulation, the central regions are approximately spherical with turbulent fluctuations in the density field. As also reported by Turk et al. (2012), there is no disk during this stage of the evolution.

Following the evolution beyond the first peak is not straightforward, as the Jeans length always needs to be resolved with at least 4 cells to avoid artificial fragmentation (Truelove et al. 1997), thus requiring more and more refinement levels as collapse proceeds. The latter can be avoided by replacing the high-density gas with sink particles, or by turning on an adiabatic equation of state at high densities. Latif et al. (2013c) followed the latter approach to pursue the subsequent evolution for five free-fall times. As shown in Fig. 5, disk structures can be clearly recognized at the end of the simulation both in the simulations with and without the turbulence subgrid-scale model. In one of the simulations, fragmentation has already occured, while most of the runs show a central massive object with approximately $10 \mathrm{M}_{\odot}$. Previous simulations employing a lower resolution per Jeans length indeed followed the evolution for longer times, indicating that fragmentation is expected to occur in the majority of these systems (Stacy et al. 2010, Clark et al. 2011; Greif et al. 2011).

As for the properties of these disks at the final stage of the simulation, we note that high accretion rates of $10^{-3}-10^{-2} \mathrm{M}_{\odot} \mathrm{yr}^{-1}$ are found in the central $100 \mathrm{AU}$, with considerable 


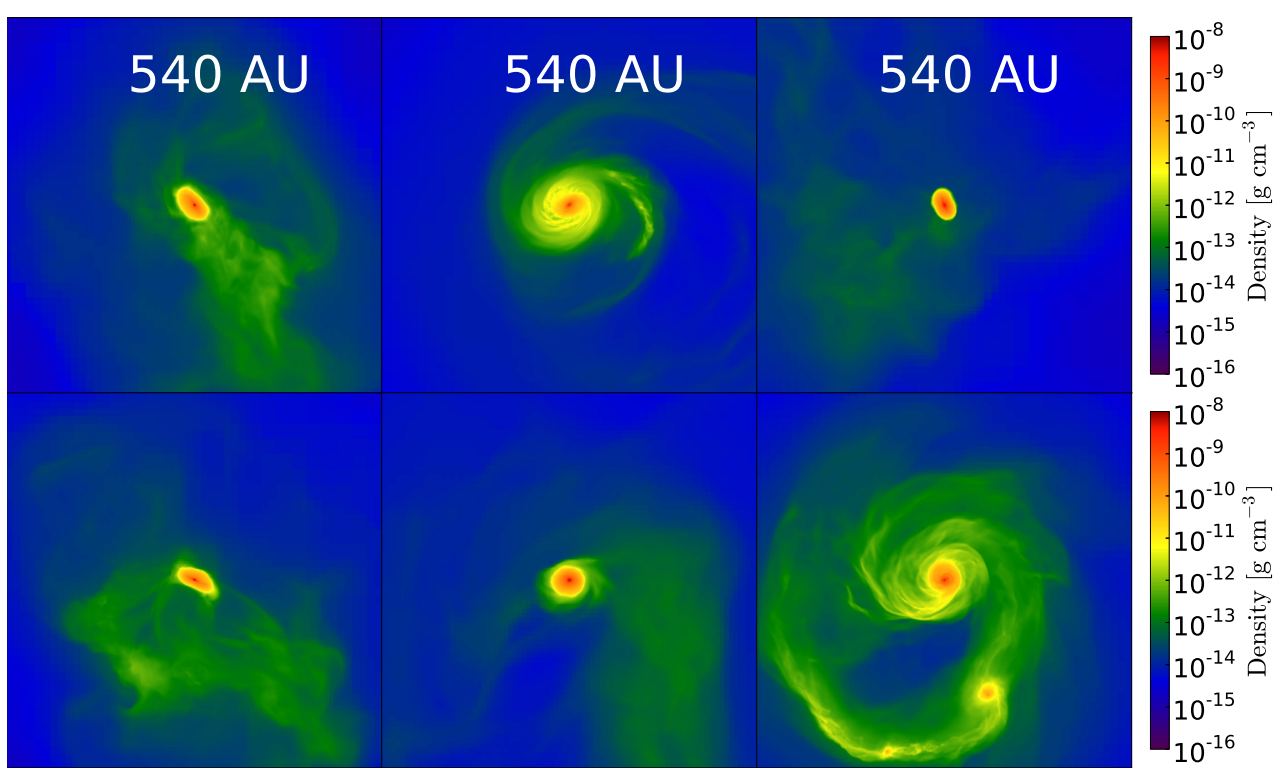

Fig. 5 Projections of the gas density in the central $540 \mathrm{AU}$ for three different halos approximately 40 years (5 free-fall times) after reaching the highest refinement level. The top panel shows purely hydrodynamical simulations, while the simulations in the bottom panel include the subgrid-scale turbulence model by Schmidt et al. (2006). Figure by Latif et al. (2013c).

spatial fluctuations as expected in a real system. On the other hand, the rotational velocities range from a few up to $10 \mathrm{~km} / \mathrm{s}$, while the radial infall velocities are of order $1 \mathrm{~km} / \mathrm{s}$.

While most of the evolution depends only on the initial conditions and the subsequent dynamics, the three-body $\mathrm{H}_{2}$ formation rate has been a major uncertainty until recently, as the rates derived by different groups (e.g. Palla et al. 1983, Abel et al. 2002, Flower \& Harris 2007) showed differences by about three orders of magnitude, implying significant uncertainties for the densities at which the transition to the fully molecular state is expected to occur. A detailed description of these uncertainties was provided by Glover et al. (2008), and their implications in 3D simulations have been explored by Turk et al.(2011). Since recently, a new accurate determination of this rate is however available from quantum-molecular calculations by Forrey (2013) which considerably reduces the uncertainties discussed here.

The impact of this rate compared to the previous rates employed in the literature has been explored by Bovino et al. (2014) in three cosmological halos using the chemistry package $\mathrm{KROM}^{8}$ (Grassi et al. 2014). A representative example is given here in the top panel of Fig. 6. For the halo shown here, the resulting $\mathrm{H}_{2}$ fraction lies inbetween the simulations based on Abel et al. (2002) and Palla et al. (1983). We however note that in some cases, the result can be closer to one of these two. The same is also true for the abundance of atomic hydrogen. The abundances of the electrons and $\mathrm{H}^{-}$are lower compared to the other simulations, which is likely a result of the different dynamical evolution as a result of the new rates. We note that this behavior varies strongly from halo to halo without a clear trend.

The impact of the chemical evolution on the dynamics is illustrated in the bottom panel of Fig. 6 We note that central quantities like the gas density have a very small dependence on the rates considered here. The gas temperature shows only minor differences for most

\footnotetext{
8 http://kromepackage.org/
} 

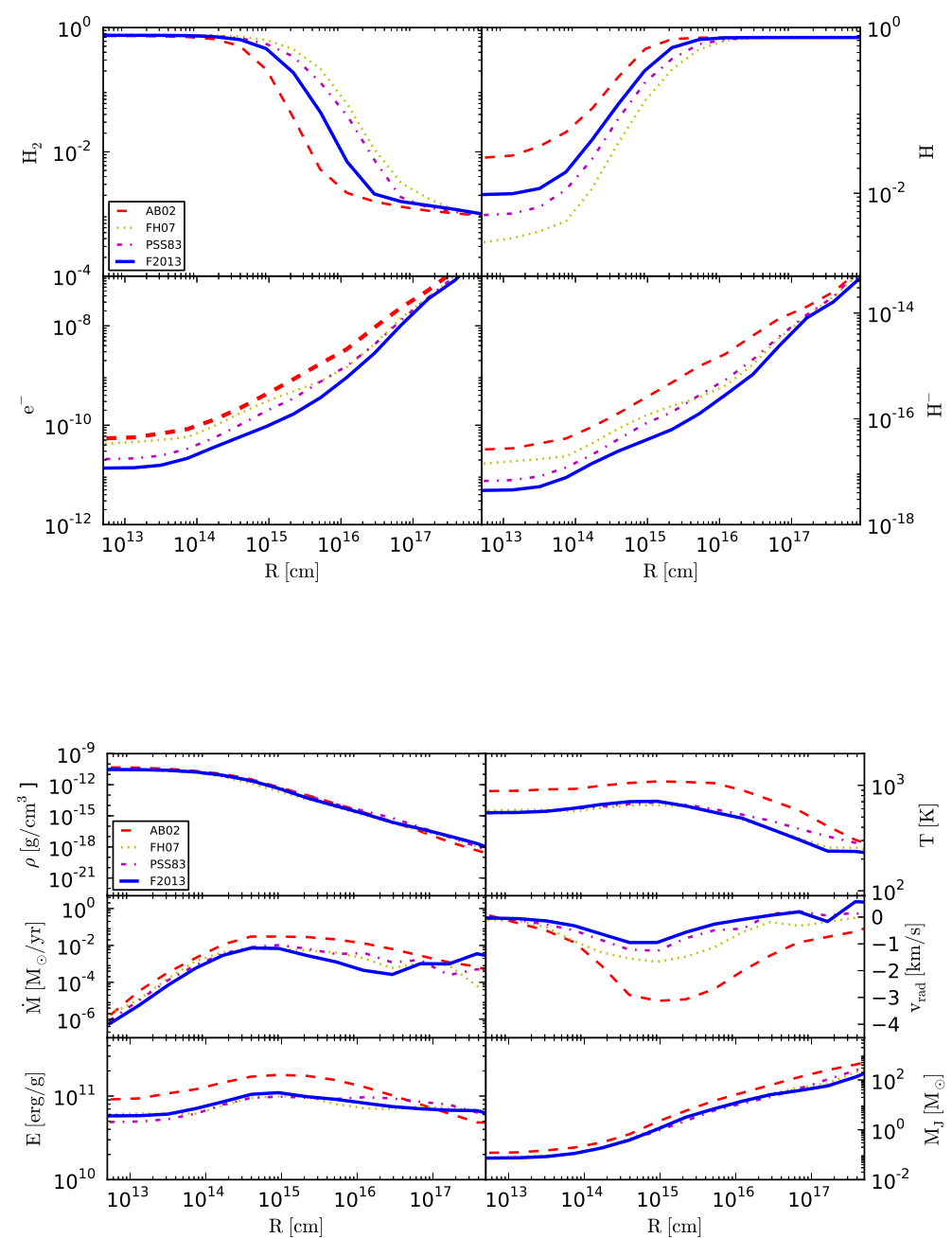

Fig. 6 A comparison of different three-body $\mathrm{H}_{2}$ formation rates based on Palla et al. (1983), Abel et al. (2002), Flower \& Harris (2007) and Forrey (2013). Figures by Bovino et al. (2014). Top panel: Shown is the $\mathrm{H}_{2}$ abundance (top left), the electron abundance (bottom left), the atomic hydrogen abundance (top right) and the abundance of $\mathrm{H}^{-}$(bottom right). Bottom panel: Shown is the gas density (top left), accretion rate (middle left), specific energy (bottom left), gas temperature (top right), radial velocity (middle right) and the Jeans mass (bottom right).

of the rates we explored, but the simulation based on Abel et al. (2002) appears to have a clearly enhanced temperature compared to the other cases. The radial velocity depends rather sensitively on the adopted three-body rate and is again larger for Abel et al. (2002). The same is true for the resulting accretion rates. The Jeans mass, on the other hand, is very similar in the cases considered here, with a small enhancement for the rate of Abel et al. 
(2002 9 We however note that relevant fluctuations in these quantities are still expected due to locally varying initial conditions.

We further note that the impact of additional physical processes can be expected to be relevant during primordial star formation. Magnetic fields are expected to form rapidly via the small-scale dynamo during the initial collapse (Schleicher et al. 2010a; Sur et al. 2010, Schober et al. 2012, Sur et al. 2012; Turk et al. 2012), and subsequent ordering may occur as a result of large-scale dynamos in the accretion disk (Pudritz \& Silk 1989 , Tan \& Blackman 2004, Silk \& Langer 2006). It thus needs to be determined whether jet formation can occur under realistic conditions (Machida et al. 2006). Recent studies further indicate that radiative feedback can potentially set an upper limit on the stellar masses of order $50-100 \mathrm{M}_{\odot}$ (Hosokawa et al. 2011, Susa 2013), but more realistic 3D investigations are required for a final conclusion.

\subsection{Black hole formation in massive primordial halos}

The potential pathways to the formation of SMBHs were already sketched by Rees (1984), and a detailed discussion would be largely beyond the scope of this review (see Volonteri \& Bellovary 2012, for a more general discussion). Here, we consider the formation of massive black holes as one potential outcome of the gravitational collapse in massive primordial halos in the presence of strong photodissociating backgrounds that destroy the molecular hydrogen leading to an almost isothermal collapse regulated by atomic hydrogen.

Such scenarios were put forward by Koushiappas et al. (2004), Begelman et al. (2006), Spaans \& Silk (2006), Lodato \& Natarajan (2007) and Schleicher et al. (2010b) (see as well Bromm \& Loeb 2003a, for an earlier work). The first numerical simulations following the gravitational collapse of these halos were reported by Wise et al. (2008), finding that gravitational instabilities may transport angular momentum and allow the formation of massive central objects. The thresholds required to fully dissociate the $\mathrm{H}_{2}$ were carefully investigated by Shang et al. (2010), while Regan \& Haehnelt (2009b) pursued the first numerical simulation exploring the evolution beyond the formation of the first peak and confirming the formation of a disk at late times. While these simulations typically employed only a moderate resolution per Jeans length, Latif et al. (2013b) demonstrated the appearance of extended turbulent structures once a high resolution per Jeans length is employed. Such turbulence can further aid the amplification of magnetic fields via the small-scale dynamo (Schleicher et al.2010a; Latif et al.2013d).

A central question is indeed how often the right ambient conditions exist in order to allow for such a close-to-isothermal collapse as required here. While a first investigation by Dijkstra et al. (2008) indicated that their abundance is likely sufficient to explain the observed population of SMBHs, more recent results (Agarwal et al. 2012, Johnson et al. 2013b) suggest that appropriate conditions typically occur for at least one halo in a box of $1 \mathrm{Mpc}^{-3}$. It is thus highly relevant to explore if such conditions lead to the formation of massive central objects.

The latter question was explored by Latif et al. (2013a) with the cosmological hydrodynamics code ENZO (O'Shea et al. 2004; Bryan et al. 2014). Their simulation setup started from cosmological initial conditions at $z=100$ in a box of $1 h^{-1} \mathrm{Mpc}$, with a root grid of $128^{3}$, two initial nested grids of the same resolution centered on the most massive halo of $\sim 10^{7} \mathrm{M}_{\odot}$, as well as 27 additional refinement levels. The refinement level adopted

\footnotetext{
9 Based on the Forrey (2013) calculation, the chemical uncertainties are thus strongly reduced.
} 


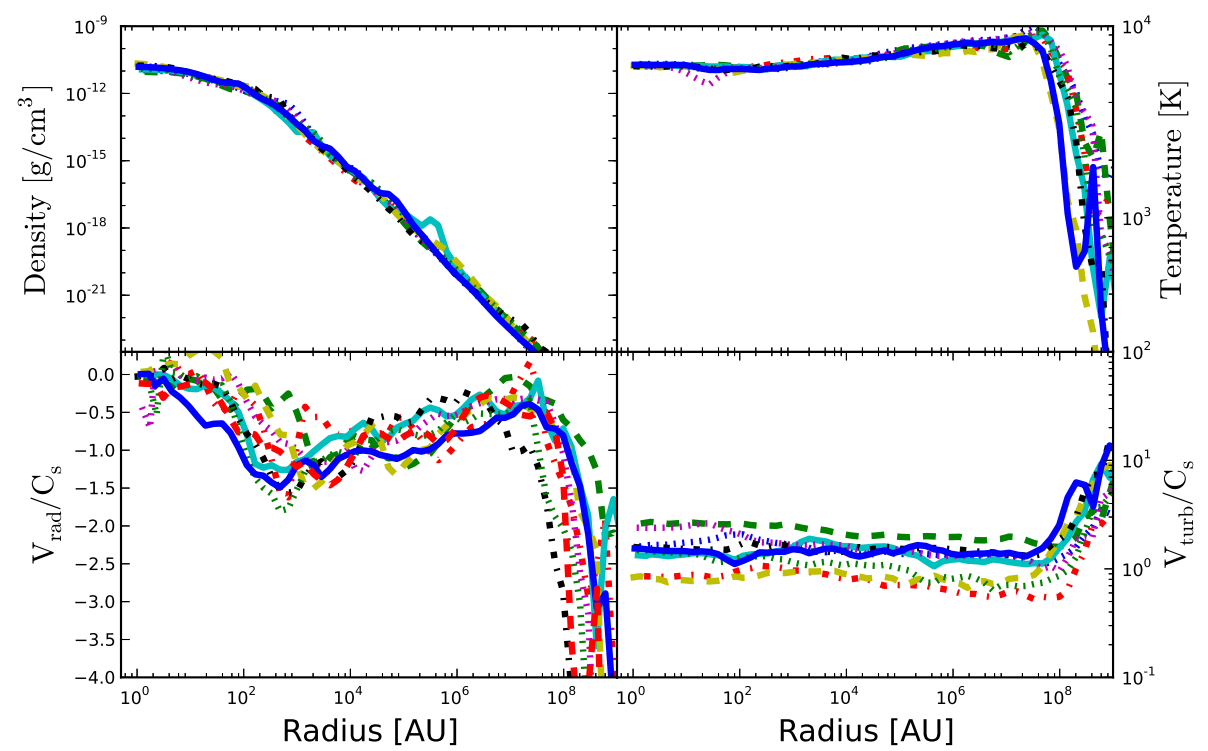

Fig. 7 The initial collapse in primordial massive halos. Shown are radial profiles of the gas density (top left), the radial velocity relative to the sound speed (bottom left), the gas temperature (top right) and the turbulent velocity normalized relative to the sound speed (bottom right). Figure by Latif et al. (2013a).

here ensured a minimum resolution of at least 64 cells per Jeans length. The simulations included primordial chemistry in the presence of a strong photodissociating background, parametrized via $J=J_{21} \cdot 10^{-21} \mathrm{erg} \mathrm{cm}^{-3} \mathrm{~s}^{-1} \mathrm{~Hz}^{-1} \mathrm{sr}^{-1}$, with $J_{21}=1000$. The simulations further included the subgrid-scale turbulence model by Schmidt et al. (2006). In order to follow the evolution beyond the first peak, the equation of state was adiabatic for densities higher than $10^{14} \mathrm{~cm}^{-3}$. A total of nine such simulations has been pursued employing a different seed for the initial conditions.

The initial state of the simulation when reaching the highest refinement level is shown in Fig. 7. The gas density follows the expected isothermal profile with $\rho \propto r^{-2}$, which flattens on the highest refinement level on scales comparable to the Jeans scale. The gas temperature is shock heated to $\sim 10^{4} \mathrm{~K}$ when falling onto the halo and then remains approximately constant during the collapse, as the atomic hydrogen cooling acts as a thermostat. The radial velocity normalized in terms of the sound speed increases during the initial infall, and subsequently becomes approximately constant. The infall appears enhanced on scales of $100 \mathrm{AU}$, potentially due to the central mass, and decreases on smaller scales where the gas is not self-gravitating. Also the turbulent velocity appears approximately constant throughout the collapse, implying that turbulence is continuously regenerated via accretion shocks and shear flows.

In order to study fragmentation and the accretion onto the central object, we have followed the simulations for four free-fall times beyond the formation of the first peak. The resulting density distribution is shown in Fig. 8 for nine different halos. In all cases, the 


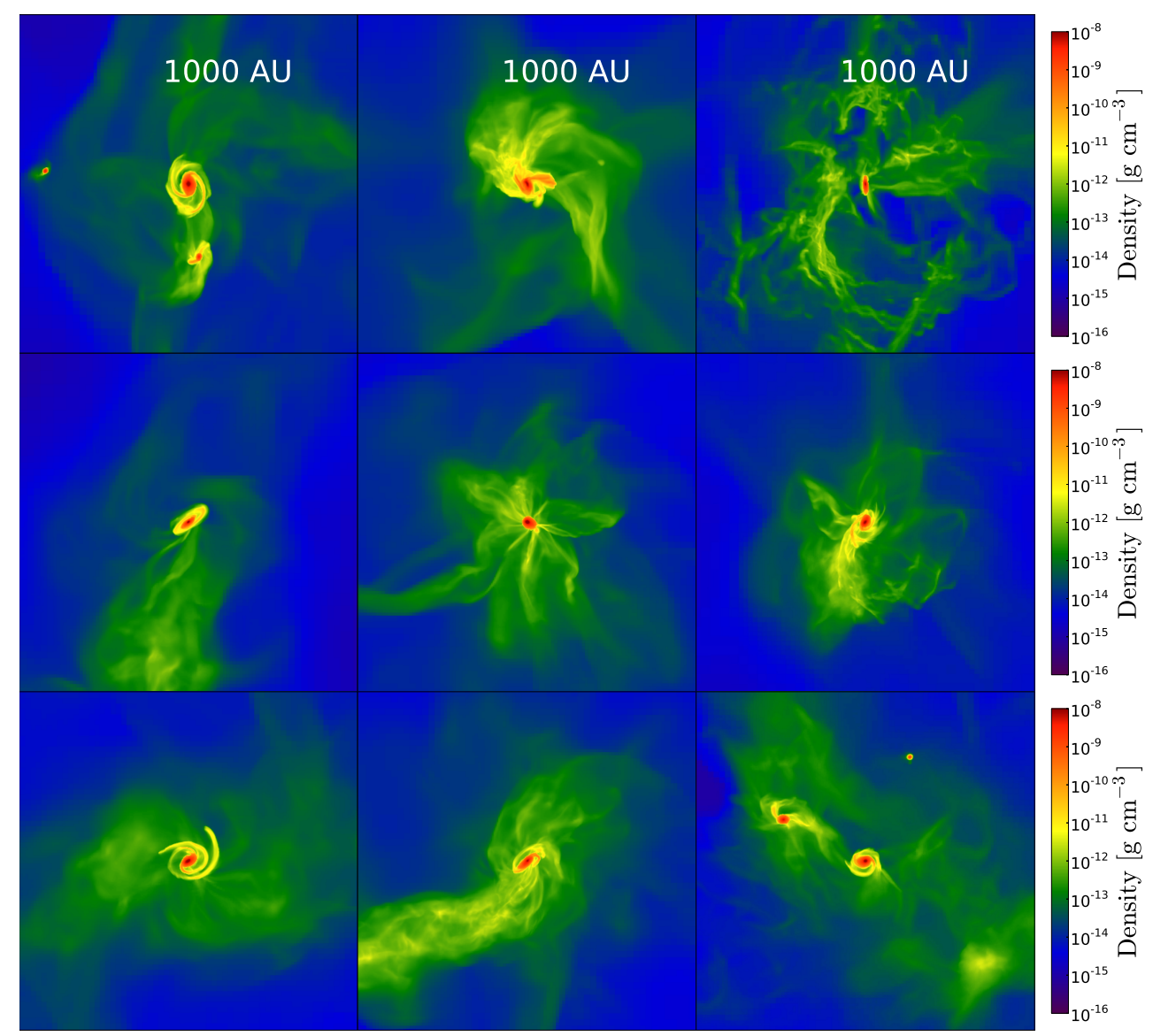

Fig. 8 Fragmentation in the central $1000 \mathrm{AU}$ for 9 different halos. Only 3 out of 9 halos fragment (one is not seen in this projection). Shown are density projections 4 free-fall times after the initial collapse. Figure from Latif et al. (2013a).

central object has already reached a mass of $\sim 1000 \mathrm{M}_{\odot}$ within the central $30 \mathrm{AU}$, with still ongoing and high accretion rates of $\sim 1 \mathrm{M}_{\odot} \mathrm{yr}^{-1}$. In these simulations, we find fragmentation in three out of nine halos (one is not visible in the projection given here). It is however possible that some of the clumps will subsequently merge, and also additional clumps may still form. The accretion occurs via self-gravitating disk structures surrounding the most massive objects. A more detailed investigation by Latif et al. (2013a) further revealed that the presence of such self-gravitating disks occured only in simulations employing the turbulence subgrid-scale model, which provided additional support for the stability of these disks, while a more filamentary accretion mode occurs in purely hydrodynamical runs.

The hydrodynamical evolution can be further assessed via Fig. 9 The figure shows the accretion rates of more than $\sim 1 \mathrm{M}_{\odot} \mathrm{yr}^{-1}$. The enclosed mass scales as the radius on larger scales, corresponding to an isothermal profile, and varies more rapidly as $r^{3}$ on small scales where the density is approximately constant. The rotational velocity in the disk is of the order several $10 \mathrm{~km} / \mathrm{s}$, while the radial velocity corresponds to $\sim 10 \mathrm{~km} / \mathrm{s}$. Similar results are also found by Regan et al. (2014). 


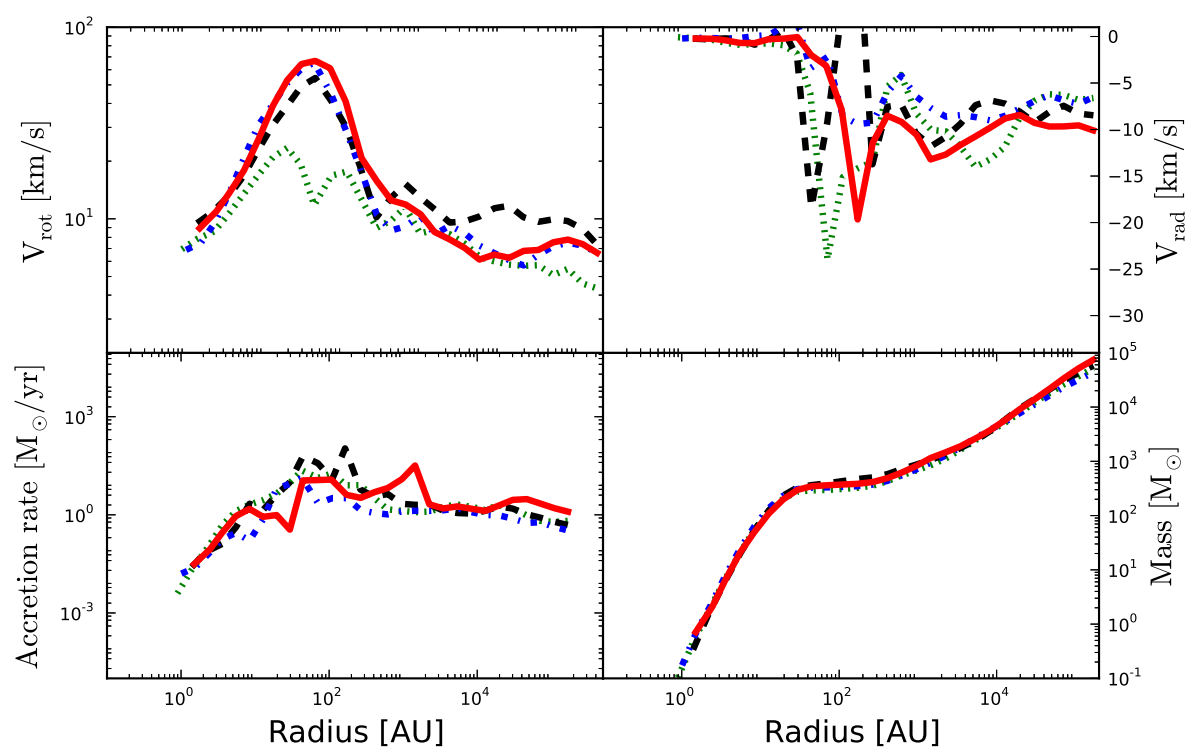

Fig. 9 Properties of the central region, 4 free-fall times after the initial collapse. Shown are radial profiles of the rotational velocity (top left), the accretion rate (bottom left), the radial velocity (top right) and the enclosed mass (bottom right). Figure from Latif et al. (2013a).

For such accretion rates, stellar evolution calculations indicate that the resulting protostar behaves as a red giant, implying a highly extended envelope and a rather cool temperature of $\sim 5000 \mathrm{~K}$ (Hosokawa et al. 2012). Considerations of the typical contraction timescales in the star indicate that such states can be maintained as long as the accretion rate is high, implying that radiative feedback is weak and leading to typical masses of $\sim 10^{5} \mathrm{M}_{\odot}$ for the resulting protostars (Schleicher et al. 2013). Even assuming that the protostars contract faster and reach the main sequence, the resulting feedback is however likely not sufficient to overcome the accretion rates (Omukai \& Inutsuka 2002, Johnson et al.2013a). It therefore appears that the formation of very massive objects is feasible. These may collapse via general-relativistic instabilities to become SMBHs (Shapiro \& Teukolsky 1986).

The black holes forming in these early stages may later become the supermassive black holes observed at $z \sim 6-7$ (Fan et al. 2001, 2003; Mortlock et al. 2011), the supermassive black holes observed in galactic centers (e.g. Magorrian et al. 1998, Häring \& Rix 2004). The feedback of such black holes may have a significant impact both on the evolution of galaxies (Somerville et al. 2008, Silk 2013) as well as on the evolution in galaxy clusters. The latter will be explained in further detail in 6 of this manuscript. 


\section{Galaxy clusters: Self-similar model}

Galaxy clusters, the largest and most massive objects in our Universe, form from the smaller units into a sequence of mergers. In this Section we briefly introduce the main predictions of the self-similar model (Kaiser 1986) which, based on relatively simple assumptions, is able of estimating the main ICM properties and important correlations between them.

The self-similar model relies on several basic assumptions. On the one hand, an EdS background cosmology and a power-law shape for the power spectrum of fluctuations are assumed. These conditions imply that there is not a characteristic scale of collapse. On the other hand, since gravity is supposed to be the unique driver of halo collapse and gas heating, there are not additional characteristic scales introduced in the process of cluster formation. This model also assumes clusters to have spherical symmetry and to be in HE (e.g. Borgani et al. 2008, see also Kravtsov \& Borgani 2012 for a recent review and extensions of this model).

Under these assumptions, the mass inside a spherical region of radius $R_{\Delta_{c}}$ enclosing a mean density equal to $\Delta_{c} \rho_{c}(z)$ at redshift $z$ is given by $M_{\Delta_{c}}=(4 \pi / 3) \Delta_{c} \rho_{c}(z) R_{\Delta_{c}}^{3}$, where $\rho_{c}(z)=\rho_{c 0} E^{2}(z)$ is the critical cosmic density at $z$, being $E(z)$ given in Eq. 6 and $\rho_{c 0}$ the critical density at $z=0$. Therefore, the cluster radius scales as $R_{\Delta_{c}} \propto M_{\Delta_{c}}^{1 / 3} E^{-2 / 3}(z)$. In addition, if the condition of $\mathrm{HE}$ is valid and the gas in clusters is distributed in a similar way to the DM, then it is satisfied that $k_{B} T \propto M_{\Delta_{c}} / R_{\Delta_{c}}$, which can be used to include in the above relation the dependence on the ICM temperature as

$$
M_{\Delta_{c}} \propto T^{3 / 2} E^{-1}(z) .
$$

This equation can now be used to derive additional scaling relations between different X-ray observables. For instance, the X-ray luminosity goes like the product of the emissivity and the cluster volume, that is, $L_{X} \propto \rho_{\text {gas }}^{2} \Lambda(T, Z) R_{\Delta_{c}}^{3}$, where $\rho_{\text {gas }}$ is the gas density and $\Lambda(T, Z)$, which depends on the gas temperature and metallicity, is the cooling function associated to a particular emission process. The X-ray emission of the ICM plasma is mainly contributed by thermal Bremsstrahlung and line emission. At high temperatures $(T \gtrsim 2 \mathrm{keV})$, where thermal Bremsstrahlung dominates, the cooling function goes like $\Lambda(T) \propto T^{1 / 2}$ (e.g. Sarazin 1986; Peterson \& Fabian 2006) and, therefore, $L_{X}$ scales with temperature as

$$
L_{X} \propto M_{\Delta_{c}} \rho_{c} T^{1 / 2} \propto T^{2} E(z) .
$$

For $T \lesssim 2 \mathrm{keV}$, however, the dependence on temperature is more intricate because line emission becomes more important than the free-free radiation and, as a consequence, the above relation has to be adjusted by accounting for the metal contribution.

An additional key quantity describing the ICM thermodynamics is the entropy (Voit 2005 ) which is usually defined as $K=\mathrm{k}_{\mathrm{B}} T n_{\mathrm{e}}^{-2 / 3}$, with $n_{\mathrm{e}}$ being the electron number density. Therefore, for pure gravitational heating the entropy scales as

$$
K_{\Delta_{c}} \propto T E(z)^{-4 / 3} \text {. }
$$

It is important to stress that the shape of the above relations and their $z$-dependence are a natural consequence of both the particular assumptions of the self-similar model and the redshift dependence of $\Delta_{c} \rho_{c}(z)$ associated to the assumed SO mass definition (Kravtsov \& Borgani 2012). Therefore, given that the standard cosmological model only introduces minor departures from self-similarity, observational deviations from these predictions can be used to determine the effects of additional physical processes other than gravity. 
In general, hydrodynamical simulations including only the effects of gravity (e.g. Navarro et al. 1995, Eke et al. 1998; Nagai et al. 2007b) are able to reproduce the shape of the above $\mathrm{X}$-ray scaling relations. However, as we discuss below, a number of observations show some important deviations from these predictions, indicating an additional contribution from nongravitational processes.

\subsection{Observational deviations from self-similarity}

A number of X-ray observations point against the simple self-similar scenario, especially at the scale of small clusters and groups. Indeed, galaxy clusters observations have confirmed that, despite the simplicity and the important predictions provided by the adiabatic model just described, there are still some important issues that deserve a further investigation:

- The cooling flow problem. At high temperatures, when the free-free radiation dominates the ICM X-ray emission, the characteristic time scale of the gas to cool down can be written as $t_{\text {cool }} \simeq 6.9 \times 10^{10} \mathrm{yr}\left(n_{e} / 10^{-3} \mathrm{~cm}^{-3}\right)^{-1}\left(T / 10^{8} K\right)^{1 / 2}$ (e.g. Sarazin 2008). According to this expression, as the gas temperature decreases, gas cooling becomes faster. In addition, given its dependence on the gas density, whereas $t_{\text {cool }}$ in outer cluster regions is usually longer than the age of the Universe, it can reach much shorter values $\left(t_{\text {cool }} \sim 10^{8}-10^{9} y r\right)$ in denser, central regions of cooling core clusters.

Some of the main features of these cooling core clusters are the following (see Sarazin 1986 for a review and references therein): (i) they have X-ray surface brightness with very high central values; (ii) their gas temperature, which is very low at the center, increases with radius; (iii) within the cooling core regions, $t_{\text {cool }}$ is lower than the Hubble time; and (iv) they show an increasing iron abundance towards the interior. These general features are detected in a considerable number of clusters which are the so-called cool core clusters (CC). In general, observations report that these properties are most often found in dynamically relaxed clusters (e.g. Fabian et al. 1994, Chen et al. 2007).

These observations led to the classical cooling flow model (e.g. Sarazin 1986, 1988). This model is based on the assumption that there is not a heating source to compensate the gas radiative cooling. Therefore, the gas cools and falls inward subsonically, eventually reaching very low central temperatures. When these temperatures go below the characteristic X-ray emitting values, strong emission lines are produced.

However, the well-known cooling flow problem stems from the observation of several facts: (i) the expected cooling rates predict emission lines stronger than observed; (ii) the ratio between the central and mean cluster temperatures only remains at a factor $\sim 1 / 3$ (e.g. Peterson et al.2003); (iii) the mass deposition rates, the amounts of cool gas and the star formation rates are generally smaller than estimated from the expected cooling rates (e.g. Edge \& Frayer 2003). These discrepancies, which imply the existence of a central heating source, gave rise to the well-known cooling flow problem: which mechanism (or mechanisms) prevents the intra-cluster gas from cooling down to low temperatures?

- Self-similar scaling relations? Contrary to what is expected from pure gravitational models, early X-ray observations of galaxy clusters demonstrated that observational scaling laws do not scale self-similarly (see Giodini et al. 2013, for a recent review).

The first indication of self-similarity breaking was the $L_{X}-T$ relation (e.g. Markevitch 1998; Allen et al. 2001; Ettori et al. 2004; Pratt et al. 2009, Maughan et al. 2012), for 

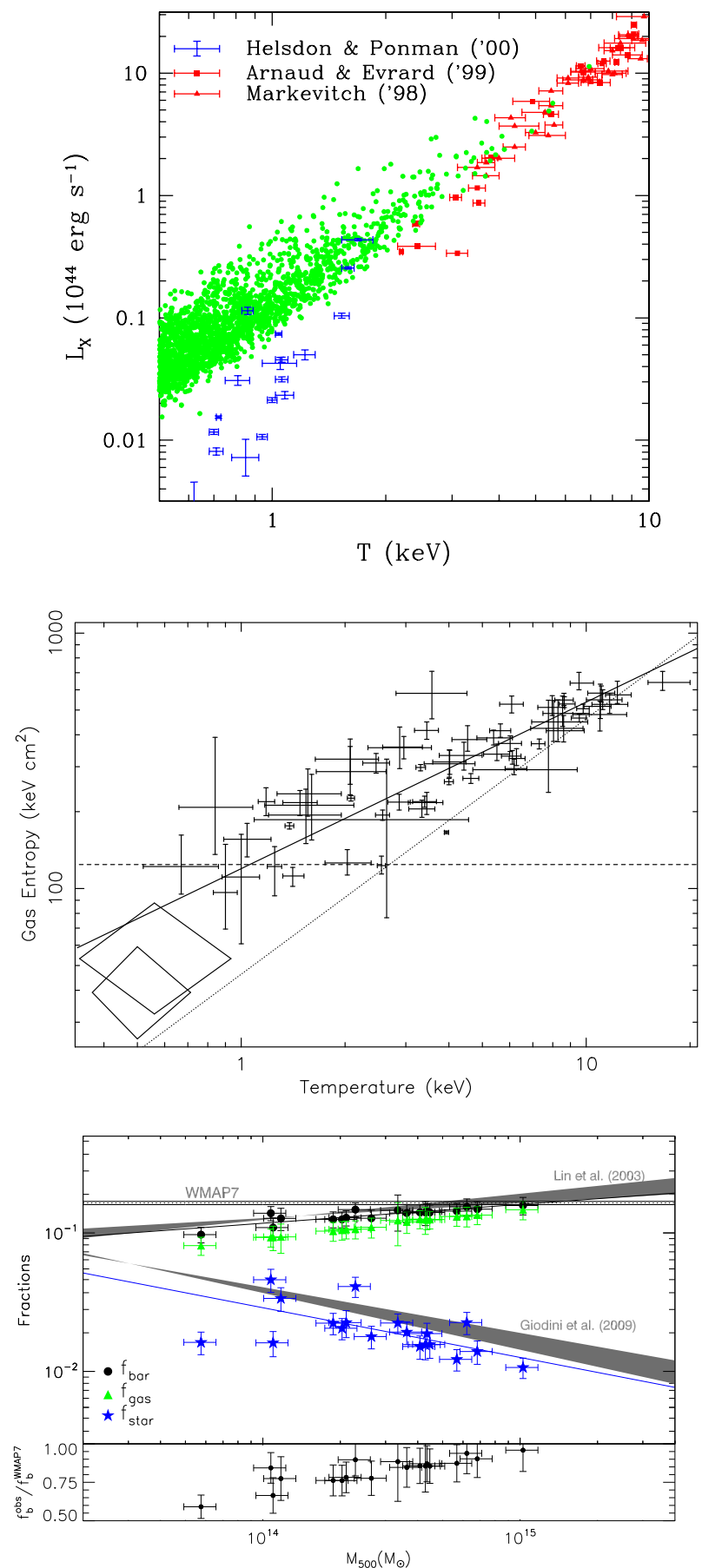

Fig. 10 Top panel: Comparison of the $L_{X}-T$ relation as obtained from different $X$-ray observational samples and from the radiative simulations by Borgani et al. (2004) (green dots). These simulations assume null metallicity and include cooling, star formation and SN feedback. Figure from Borgani et al. (2004). Middle panel: Relation between gas entropy and temperature for a sample of galaxy clusters and groups. The solid line represents the observational relation, whereas the dotted line stands for the self-similar prediction. Figure from Ponman et al. (2003). Bottom panel: Observations of the baryonic, gas and stellar mass fractions as a function of total mass. The power-law fits obtained for the baryonic and stellar mass fractions are given by the black and blue solid lines, respectively. For comparison, the corresponding best-fits derived by Lin et al. (2003) and Giodini et al. (2009) are also included. The lower panel shows the ratio between observational and WMAP-7 baryon mass fraction. Figure from Laganá et al. (2011). 
which observations report a steeper slope $(\approx 3)$ than the expected self-similar value of 2 (e.g. Markevitch 1998; Osmond \& Ponman 2004), a departure that becomes even larger for systems with $k_{\mathrm{B}} T \lesssim 3.5 \mathrm{keV}$ (Ponman et al. 1996, Balogh et al. 1999, Maughan et al. 2012). In general, the observed $L_{X}-T$ relation shows a relatively large scatter (e.g. Pratt et al. 2009), which is mainly due to both the strong emission associated to CC clusters and to unrelaxed systems for which the HE condition is not a good approximation (e.g. Maughan et al. 2012). A common practice to reduce this scatter consists in excluding either cluster cores (e.g. Markevitch 1998, Pratt et al. 2009), or CC systems (e.g. Arnaud \& Evrard 1999). As an example, the top panel of Fig. 10 shows a comparison of the luminosity-temperature relation as obtained from different X-ray observational samples (Arnaud \& Evrard 1999; Markevitch 1998, Helsdon \& Ponman 2000) and from the radiative simulations with $\mathrm{SN}$ feedback by Borgani et al. (2004). As we can see, numerical results are consistent with observations for $k_{B} T \gtrsim 2 \mathrm{keV}$. However, the agreement is not so encouraging at $k_{B} T \lesssim 2 \mathrm{keV}$, where simulations may include additional or more efficient feedback processes to further reduce the X-ray emission.

Consistently with the $L_{X}-T$ scaling, the relation between X-ray luminosity and mass is also steeper than expected from self-similarity (e.g. Reiprich \& Böhringer 2002, Chen et al. 2007). As shown in the bottom panel of Fig. 10, a possible reason for this may be related with the observations of an increasing trend of the gas mass fraction with total mass (e.g. Balogh et al. 2001; Lin et al. 2003, Sanderson et al. 2003, Vikhlinin et al.2006, Pratt et al. 2009, Dai et al.2010; Laganá et al.|2011). The lower gas content observed in low mass systems would generate a lower X-ray emission and, therefore, a steepening of the scaling laws.

As can be inferred from the middle panel of Fig. 10 observations of central regions of small galaxy clusters and groups report higher entropy values than expected from pure gravitational predictions, thereby generating a flattening of the observed $K-T$ relation (Ponman et al. 2003). This increase of the gas entropy in low mass systems prevents the gas from falling into the center, reducing the central amounts of gas and, as a consequence, leading to the steepening of the observed $L_{X}-T$ relation (e.g. Evrard \& Henry 1991; Tozzi \& Norman 2001; Borgani et al. 2001, Voit et al. 2002).

As for the total mass-temperature relation, observations generally report a self-similar behavior for massive galaxy clusters (e.g. Arnaud et al. 2005; Vikhlinin et al. 2009), whereas a slightly steeper slope is obtained for smaller objects (e.g. Arnaud et al. 2005, Sun et al. 2009, see also Kettula et al.2013 for a recent weak lensing calibration of this relation). In addition, given its small intrinsic scatter, which is mainly due to the existence of substructures (e.g. O'Hara et al. 2006, Yang et al. 2009), the $M_{\mathrm{tot}}-T$ relation turns out to be extremely useful for cosmological applications with galaxy clusters.

- Temperature and entropy radial profiles. Independent X-ray observations have confirmed that the ICM temperature distribution is not isothermal. Indeed, as it is shown in the left panel of Fig. 11 the cluster temperature radial profiles are characterized by negative gradients at $r \gtrsim 0.1 R_{180}$ (e.g. Piffaretti et al. 2005, Pratt et al. 2007), being nearly self-similar out to the most external regions (e.g. Markevitch 1998; De Grandi \& Molendi 2002, Vikhlinin et al. 2005). However, the shape of these profiles in inner cluster regions partially depends on the dynamical state of the considered systems: dynamically relaxed clusters generally show decreasing temperature profiles towards the core, while unrelaxed systems show, instead, different patterns. In principle, while gas radiative cooling might be able to generate low temperatures in the central regions of CC 

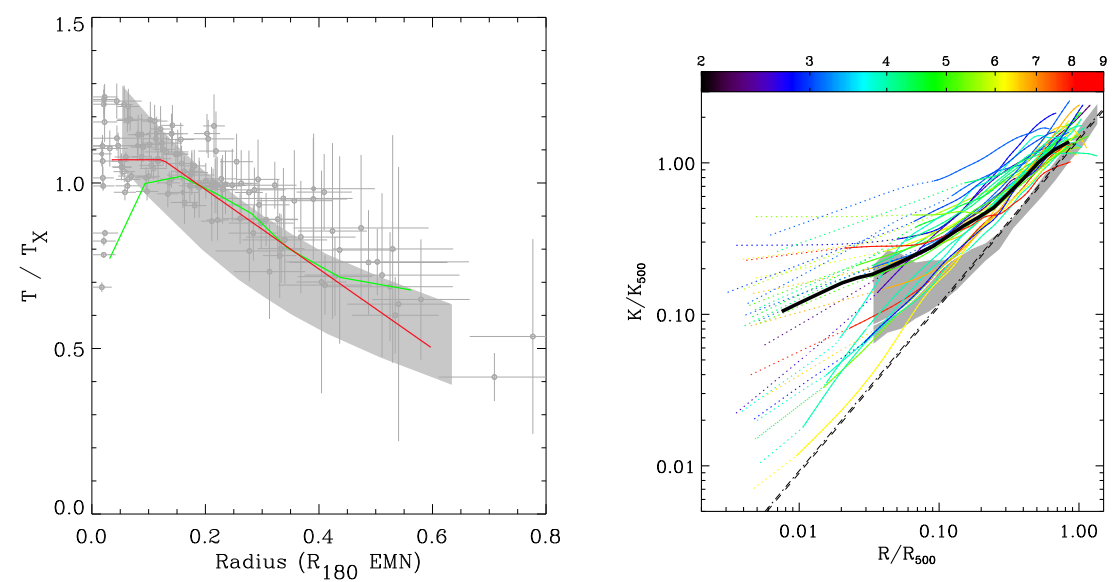

Fig. 11 Left panel: Comparison of the dimensionless temperature profiles from XMM-Newton observations of 15 nearby clusters by Pratt et al. (2007) (dots with error bars) with the average profiles from ASCA (grey band, Markevitch 1998), and the observations of cooling core clusters from BeppoSAX (green line, De Grandi \& Molendi 2002) and Chandra (red line, Vikhlinin et al. 2005). Figure from Pratt et al. (2007). Right panel: Comparison of the scaled entropy profiles of the REXCESS sample (colored lines according to the temperature of the clusters, Pratt et al. 2010) with the theoretical results from the non-radiative simulations by Voit (2005). The thick black line represents the median of all the observed profiles, whereas the dashed line stands for the best power law fit to the median profile of the simulated clusters. Figure from Pratt et al. (2010).

clusters, some source of energy feedback may avoid an excess of cooling, thus reducing the resulting star formation rates in these systems.

As for the entropy radial profiles, pure gravitational models predict that, in outer cluster regions, entropy scales with radius as $K \propto r^{1.1}$ (Tozzi \& Norman 2001). However, recent observations (e.g. Simionescu et al. 2011, Walker et al. 2012, Eckert et al. 2013) have reported that these profiles flatten in central cluster regions and their dependence on radius at larger radii is gentler than predicted (see right panel of Fig. 11). The particular radius at which the profiles become flat depends on a number of factors, being shorter for CC than for non cool-core (NCC) clusters (e.g. Sanderson et al. 2009, Pratt et al.2010). Moreover, while high-mass clusters show a higher mean core entropy (e.g. Cavagnolo et al. 2009) and nearly self-similar profiles in outer regions, smaller systems are characterized by shallower profiles. This distinction between low and high mass systems, which is a clear indication of the higher efficiency of non-gravitational processes on smaller objects (e.g. Cavagnolo et al. 2009; Pratt et al. 2010, Maughan et al. 2012), highlights that entropy profiles provide an important fingerprint of the different physical processes breaking cluster self-similarity (e.g. Voit et al.|2002,2003).

The existing disparity between the self-similar predictions and the observations clearly indicates that, in addition to the effects of gravity, additional processes related to the physics of baryons should be also taken into account. Therefore, additional non-gravitational processes operating within galaxy clusters and groups, such as radiative cooling, star formation and its inferred energy feedback, or AGN feedback, should interact among them in order to solve the aforementioned problems. As we discuss below, efforts to obtain a completely consistent and satisfactory model able to reproduce the observations are still ongoing (e.g. Borgani et al. 2004; Voit 2005, McCarthy et al. 2008). 


\section{The physics of the intra-cluster plasma}

Gravitational processes lead the evolution of initial density fluctuations into DM halos. However, as we review in this Section, the thermodynamics of the ICM is determined by the combined action of gravity, acting on both DM and baryonic components, and a number of non-gravitational processes such as radiative cooling, star formation and AGN feedback.

\subsection{Structure formation shock waves}

As explained in 2.3 within the hierarchical $\Lambda \mathrm{CDM}$ paradigm the formation of DM halos is driven by the gravitational collapse of initial matter density perturbations. Figure 1, which displays the formation and evolution of galaxy clusters as obtained from hydrodynamical cosmological simulations, indicates that in addition to DM (left panels), the evolution of the hot diffuse gas (middle panels) and the stellar (right panels) components are significantly connected to each other. At $z=0$, galaxy clusters are identified as high-overdense DM regions at the intersection of large matter filaments. Whereas the gas distribution follows closely the DM distribution, the pattern of the stellar component is much clumpier.

As a consequence of the hierarchical process of structure growth, clusters of galaxies often undergo major merger episodes, which are one of the most energetic phenomena in the Universe (Sarazin 1988). These mergers, associated to the collisions of proto-cluster structures of similar masses at velocities of several thousands of kilometers per second, generate shocks and compression waves in the ICM. On large scales, these hydrodynamical shocks are very efficient in releasing an important amount of the energy associated with the collision $\left(\sim 10^{61}-10^{65} \mathrm{ergs}\right)$ as thermal energy in the final system, heating and compressing the hot intra-cluster gas and, therefore, increasing its entropy (e.g. Quilis et al. 1998, Miniati et al. 2000). On smaller scales within already collapsed structures, additional weaker shocks are developed as a consequence of subhalo mergers, accretion processes, or random gas flows. These internal shocks are relevant in the thermalization of the intra-cluster gas (McCarthy et al. 2007), contributing significantly to the virialization of halos.

In addition, shocks also generate ICM turbulence and mixing (Norman \& Bryan 1999, Ricker \& Sarazin 2001, Nagai et al. 2003, Dolag et al. 2005), redistribution and amplification of magnetic fields (Roettiger et al. 1999), and are likely sources of non-thermal emission in galaxy clusters (e.g. Bykov et al. 2000). Indeed, cosmological simulations have shown that an important non-thermal pressure support in galaxy clusters is provided by ICM turbulence (e.g. Dolag et al. 2005, Vazza et al.2011), relativistic CR particles (e.g. Pfrommer et al. 2007; Vazza et al. 2012), and magnetic fields (Dolag et al.|1999). In general, none of these processes alone can regulate cooling, but they may be more efficient when AGN feedback (Voit 2011) or plasma instabilities (e.g. Sharma et al.2012) are also considered.

Therefore, it is clear that cosmological shocks leave their imprint on relevant ICM plasma properties (see Bykov et al. 2008, for a review). First, the rise of the gas entropy and thermal pressure support induced by shocks represents a natural alternative way of breaking the self-similar scaling (e.g. Bykov et al.2008, for a multi-fluid accretion shock model, derived $K \propto T^{0.8}$ ). Second, shocks surrounding mildly overdense, non-linear, non-collapsed structures, such as sheets and filaments, heat the intergalactic medium and determine the evolution of the warm-hot intergalactic medium (WHIM; e.g. Cen \& Ostriker 1999; Davé et al.2001). Finally, the diffusive shock acceleration process (DSA; e.g. Drury \& Falle 1986, Blandford \& Eichler 1987) can convert a part of the thermal population of particles into nonthermal CRs, resulting therefore in both thermal and non-thermal energy constituents (Kang 


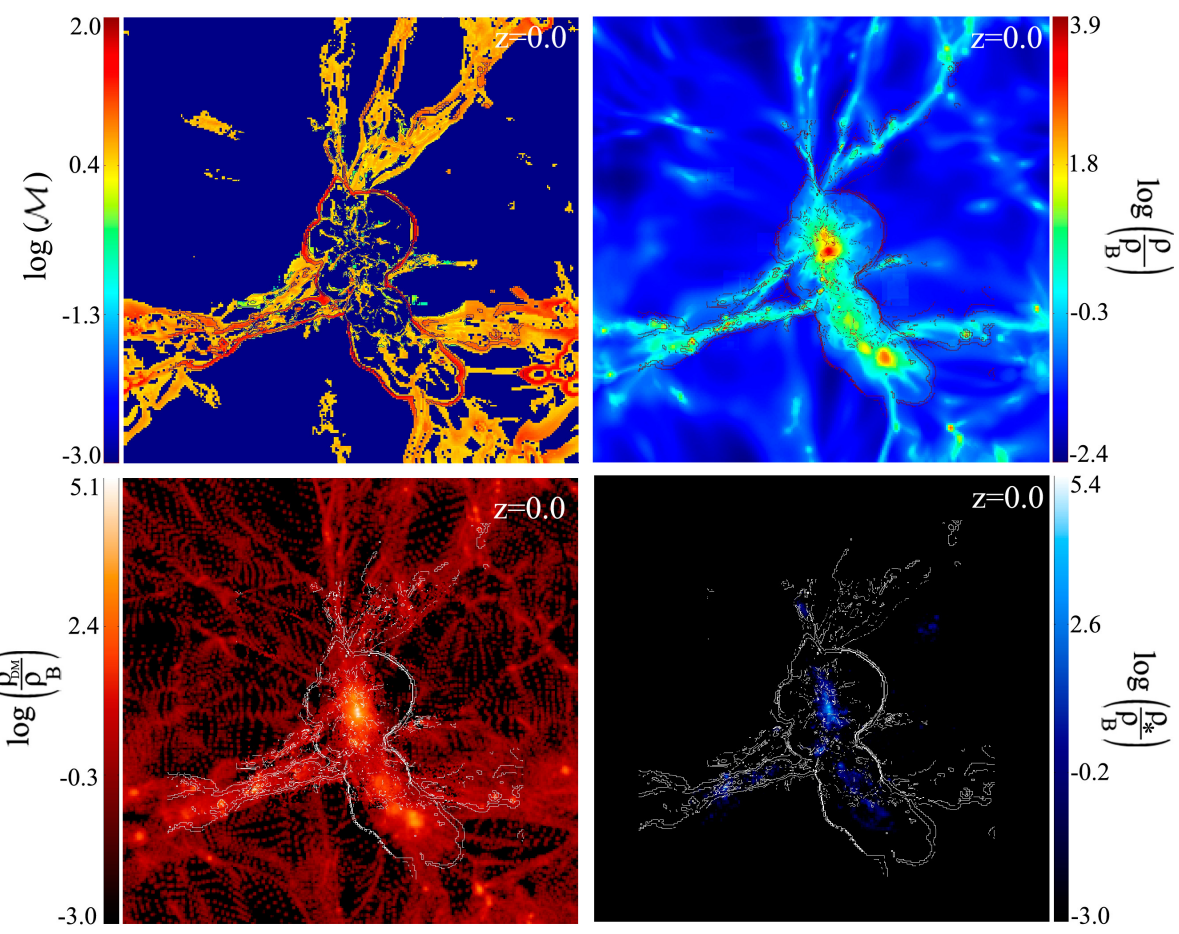

Fig. 12 Distribution of shock Mach numbers (upper left panel) and its comparison with the DM $\left(\rho_{D M} / \rho_{B}\right.$, lower left), gas $\left(\rho / \rho_{B}\right.$, upper right) and stellar $\left(\rho_{*} / \rho_{B}\right.$, lower right) overdensities at $z=0$. In all the panels, the contours of the shock waves with high Mach numbers are overplotted. Each panel represents a projection of 0.2 Mpc thickness and 64 Mpc side length. Figure from Planelles \& Quilis 2013).

et al. 2002, Kang \& Jones 2005, Kang et al. 2007). Whereas the CR electrons may be responsible of the observed radio halos (e.g. Ferrari et al. 2008, Giovannini et al. 2009) and radio relics (e.g. Ensslin et al. 1998) in clusters (e.g. Pfrommer et al. 2008), the CR protons may generate $\gamma$-ray emission.

From an observational point of view, detecting large-scale shocks is non-trivial because they take place in the periphery of galaxy clusters where the X-ray emission, due to the relatively low gas density, is weak. In spite of this, large-scale shocks have been identified in the form of radio relics in more than 30 clusters (e.g. Bonafede et al. 2009; van Weeren et al. 2009). Some merger-induced internal shocks have been also detected with Mach numbers ${ }^{10} \mathscr{M} \sim 1.5-3$ (e.g. Markevitch \& Vikhlinin 2007). From the theoretical point of view, several approaches to study shocks have been pursued using semi-analytical models (e.g. Press \& Schechter 1974; Sheth \& Tormen 1999) as well as numerical simulations based on both grid-based, single-grid (e.g. Quilis et al. 1998, Miniati et al. 2000, Ryu et al. 2003; Vazza et al. 2009) and AMR schemes (e.g. Skillman et al. 2008, Planelles \& Quilis 2013), and particle-based codes (e.g. Pfrommer et al. 2006). As an example of the results obtained from these numerical analysis, Fig. 12 shows the distribution of the shock waves detected in a cosmological AMR simulation compared with the distributions of DM, gas and stellar

\footnotetext{
10 The Mach number, which characterizes the strength of a shock, is given by $\mathscr{M}=v_{s} / c_{s}$, where $v_{s}$ is the shock speed and $c_{s}$ is the sound speed ahead of the shock.
} 
overdensities at $z=0$. This figure clearly highlights that cosmological shock waves, which occupy the whole simulated volume in a complex way, accurately trace the cosmic web. External shocks, with quasi-spherical shapes and at relatively large distances from the center of the structure where they appear, are characterized by high Mach numbers $(\mathscr{M} \gtrsim 20)$. On the contrary, within collapsed objects, weaker shocks with $\mathscr{M}<2-3$ are present, contributing significantly to the dissipation of kinetic energy (e.g. Skillman et al. 2008).

In spite of all the studies and of the long-standing debate about the use of different numerical techniques (e.g. Agertz et al. 2007), the identification of cosmological shocks still represents an issue.

\subsection{Non-gravitational processes}

'Gravitational feedback', mainly in the form of shock and compression waves, contributes to most of the intra-cluster gas heating. However, as discussed in $\$ 5.1$, the discrepancies with the self-similar model observed in small clusters and groups and in inner cluster regions, indicate the existence of additional non-gravitationally induced cooling and heating processes. In the following, we explain some of the main non-gravitational processes that need to be incorporated in hydrodynamical simulations to reproduce the self-similarity breaking.

\subsubsection{Gas radiative cooling}

Radiative cooling, which plays a major role in the ICM emissivity, can significantly contribute to break self-similarity to the observed level. To understand the role of cooling, it is useful to express the cooling time in terms of the gas entropy and temperature, which in the Bremsstrahlung regime can be approximated by (Sarazin 2008)

$$
t_{\text {cool }} \approx 17\left(\frac{K}{130 \mathrm{keV} \mathrm{cm}^{-2}}\right)^{3 / 2}\left(\frac{k_{\mathrm{B}} T}{2 \mathrm{keV}}\right)^{-1} \text { Gyrs. }
$$

Therefore, for a galaxy cluster with $k_{\mathrm{B}} T \sim 2.5 \mathrm{keV}$, the cooling time is lower than the Hubble time for an entropy of $K \lesssim 130 \mathrm{keV} \mathrm{cm}^{-2}$. This means that, the lower entropy gas within galaxy clusters will cool before, being evacuated earlier from the hot gas in cluster core regions. This low-entropy gas will be superseded by higher entropy gas coming from outer regions, thus increasing the mean gas entropy (Voit \& Bryan 2001).

Hydrodynamical simulations including radiative cooling support this prediction (e.g. Pearce et al. 2000; Muanwong et al. 2001; Davé et al. 2002, Valdarnini 2002, Kay et al. 2004, Nagai et al. 2007a). As an example, Fig. 13 shows the comparison between the mean ICM profiles of relaxed clusters derived from cosmological simulations by Nagai et al. (2007a) and the observations by Vikhlinin et al. (2006). In particular, there are two sets of simulations, one accounting only for non-radiative physics $(N R)$ and another taking into account radiative cooling, star formation and metal enrichment $(C S F)$. From the analysis of this figure we infer that, whereas the $N R$ simulations produce ICM profiles in conflict with observations, the $C S F$ runs produce a better match, at least outside cluster core regions. Specifically, at $r \gtrsim 0.5 r_{500}$, the $C S F$ runs produce both an increase in the gas entropy in accordance with the observed values, and nearly self-similar pressure profiles. Another effect of cooling is the reduction of the gas density in inner regions (see top-left panel of Fig. 13.

However radiative cooling has also some undesirable effects. As it is shown in the topright panel of Fig. 13, an unexpected effect of cooling is that of increasing the ICM temperature towards the cluster center together with the steepening of the temperature profiles. This 

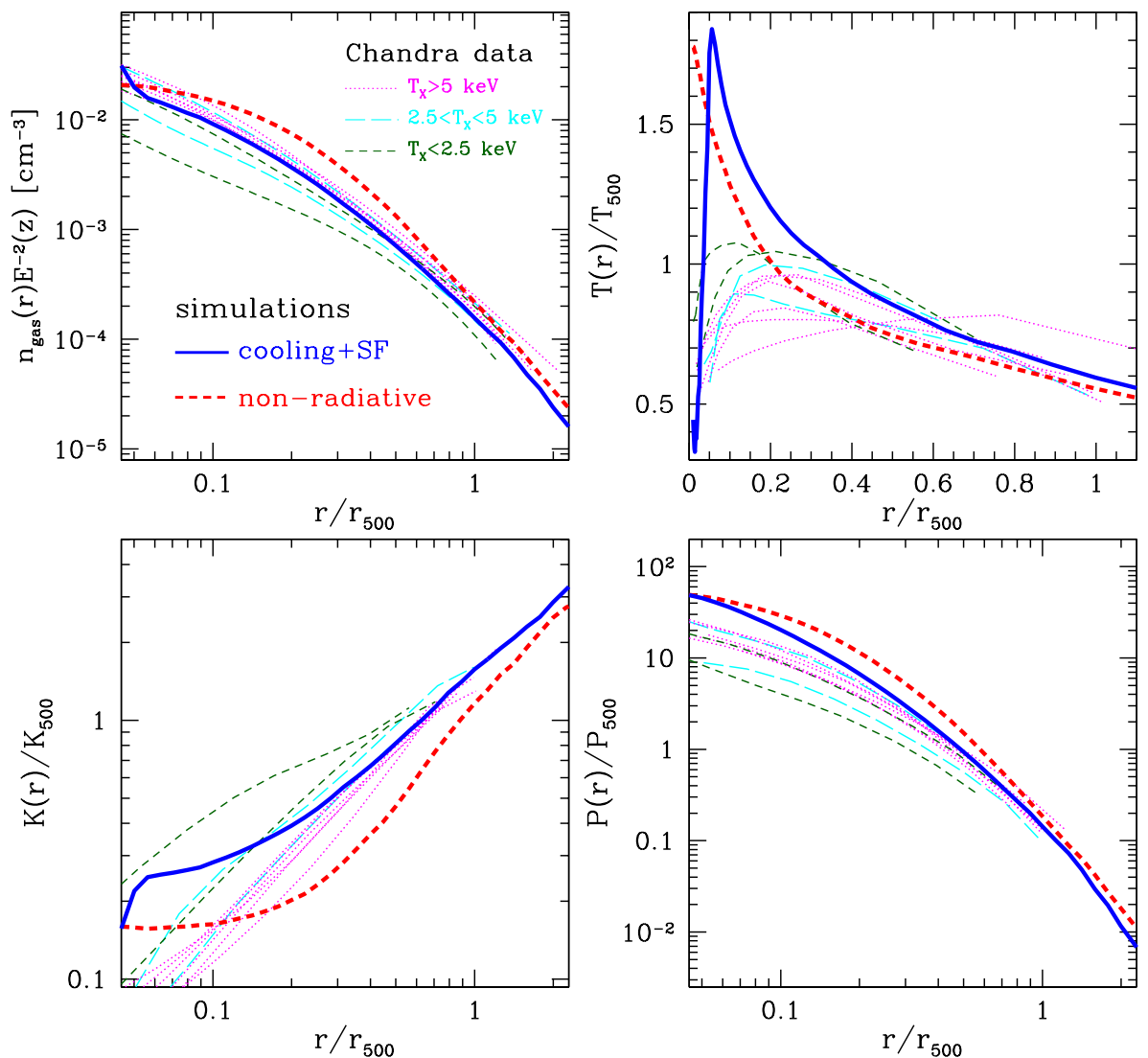

Fig. 13 From top to bottom clockwise: mean radial profiles of gas density, temperature, pressure, and entropy of relaxed clusters at $z=0$ identified in cosmological simulations. There are two sets of simulations, one non-radiative (red thick dashed lines) and another accounting for cooling $+S F$ (blue thick solid lines). The observed Chandra cluster sample by Vikhlinin et al. (2006) is used for comparison (thin lines in different colors indicating the temperature of the systems). Figure from Nagai et al. (2007a).

effect results from the dearth of central pressure support induced by cooling, which makes outer gas to fall sub-sonically to the center while being heated adiabatically. In addition, given the runaway nature of radiative cooling, it suffers from overcooling, thus transforming quite large amounts of gas into stars. In fact, radiative simulations including different forms of stellar feedback obtain that around $35-40 \%$ of the cluster baryon content is converted into stars (e.g. Nagai \& Kravtsov 2004), a value which is significantly larger than observed ( 10 - 15\%; e.g. Balogh et al. 2001; Lin et al. 2003, Gonzalez et al. 2007). In principle, these two shortcomings of cooling represent two sides of the same problem (Borgani \& Kravtsov 2011). A proper source of gas heating (or most likely, a combination of several) able of counterbalancing cooling, keeping the gas pressurized in inner regions, and controlling the star formation, may provide the solution to this complicated issue. However, as it will be discussed in the following sections, unearthing such a mechanism is non-trivial and represents nowadays an important challenge in the numerical description of clusters. 


\subsubsection{Star formation and its associated feedback}

SN explosions contribute to both the heating of the surrounding medium and the distribution of metals from star-forming regions into the hotter intra-cluster plasma. Given that SNdriven winds are a by-product of the star formation process, $\mathrm{SN}$ feedback was suggested to produce a realistic and self-regulated cosmic star formation rate (e.g. Springel \& Hernquist 2003). We note that even beyond feedback models, appropriate subgrid-scale models for hydrodynamical turbulence may be required for the description of the hydrodynamical state (e.g. Schmidt et al. 2006; Iapichino \& Niemeyer 2008, Maier et al. 2009). Moreover, given the relatively low resolutions reached by present-day cosmological simulations, the physics of the interstellar medium can not be properly described (Borgani \& Kravtsov 2011), a problem that persists even in relatively well-resolved simulations of individual galaxies (e.g. Tasker \& Tan 2009, Dobbs et al. 2010, Wang et al. 2010, Bournaud et al. 2010, see also Mayer et al.2008 for a review). Given the current limitations, stellar feedback is usually incorporated in cluster simulations via phenomenological prescriptions of star formation and SN heating (e.g. Braun \& Schmidt 2012) at moderately low resolutions.

In general, cosmological simulations show that $\mathrm{SN}$ feedback can help in partially offsetting radiative cooling, flattening the temperature profiles, and reducing the cluster stellar mass fractions (see Borgani \& Kravtsov 2011, and references therein). However, in spite of these promising results, stellar feedback alone is not efficient enough to produce the observed thermal structure of CC clusters. As an example, the left panel of Fig. 14 shows the comparison between the temperature profiles of a sample of relaxed clusters as derived from observations and from simulations including SN feedback. We see that, whereas in outer cluster regions, $r \gtrsim 0.2 R_{180}$, simulations recover quite well the observed profiles, within inner regions the agreement is not so satisfactory. The low efficiency of SN feedback in compensating the cooling properly produces additional undesirable results (e.g. Kravtsov \& Borgani 2012): in general, the levels of core entropy, although reduced, remain significantly larger than reported by observations; the BCGs have stellar masses larger than observed; and, the excessive star formation is also translated into an excessive metal production in cluster central regions (see right panel of Fig. 14).

\subsubsection{AGN feedback}

Currently, the most favored mechanism to explain the ICM self-similarity breaking and the cooling flow problem is the AGN heating resulting from gas accretion onto a central SMBH. Indeed, many cluster observations confirm the effects of AGN heating on the ICM plasma (e.g. McNamara \& Nulsen 2007, Chandran et al. 2009). First, the existence of SMBHs at the nuclei of galaxies (Magorrian et al. 1998) and the observed correlations between the BH masses and the halo and bulge properties of the host galaxies (Ferrarese \& Merritt 2000) point to an scenario in which galaxy formation and $\mathrm{BH}$ growth must proceed together. Second, as already reported by many observations (e.g. Burns 1990, Ball et al. 1993, Sanderson et al.2006), almost every dynamically relaxed CC cluster has an active central radio emitting source, which has been associated by $\mathrm{X}$-ray observations with the presence of cavities or bubbles in the X-ray emitting gas around the central galaxy (e.g. Bîrzan et al.|2004). Third, there is a clear connection between the ICM X-ray luminosity within the core of clusters and the mechanical (e.g. Bîrzan et al. 2004) and radio luminosities (e.g. Eilek 2004) of the central AGN. Another important point in favor of this heating mechanism is that AGN feedback is a self-regulated process, compensating in a natural way radiative cooling (e.g. Rosner \& Tucker 1989). This is due to the fact that the efficiency of AGN feedback is proportional to 

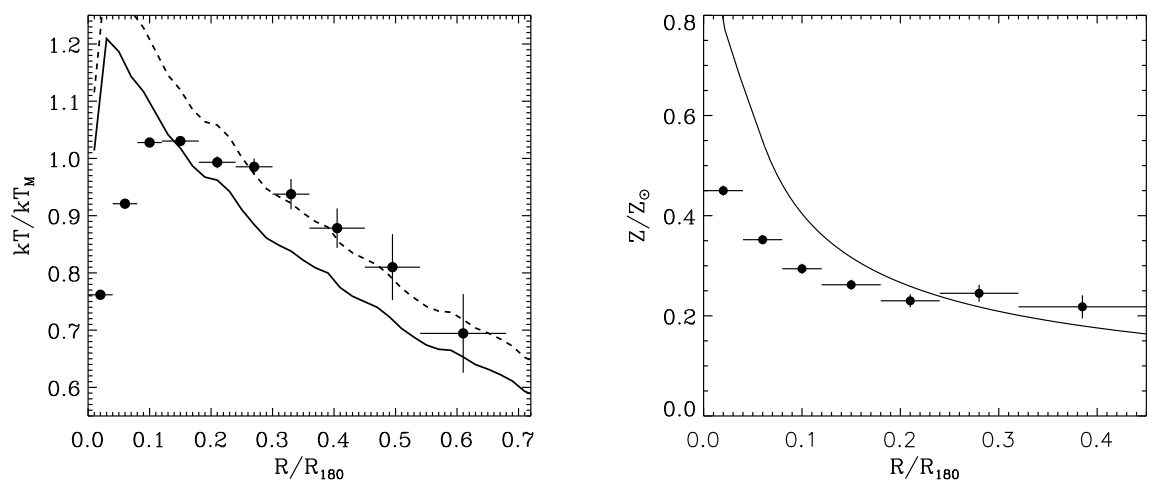

Fig. 14 Left panel: Comparison between the mean temperature profile of a sample of about 50 local $(z \lesssim 0.3)$ and hot $\left(k_{B} T_{X}>3 \mathrm{keV}\right) X M M$-Newton clusters (dots with error bars; Leccardi \& Molendi 2008b) and the mean profile obtained from cosmological simulations including radiative cooling, star formation and SN feedback (solid line; Borgani et al. 2004). The dashed line stands for the mean simulated profile rescaled by $10 \%$. Figure from Leccardi \& Molendi 2008b. Right panel: Comparison of the mean metallicity profile for the same sample of XMM-Newton clusters (dots with error bars) with the one derived by Fabjan et al. (2008) from simulations performed with the SPH code GADGET-2 (Springel 2005) assuming the chemical enrichment model by Tornatore et al. (2007). Figure from Leccardi \& Molendi (2008a).

the rate at which the central SMBH accretes intra-cluster gas while radiative cooling takes place. Therefore, if the feedback efficiency is too large, the ICM is naturally over-heated and the gas accretion is reduced. On the contrary, if the gas accretion rate is too low, the intra-cluster plasma cools faster, the accretion rate onto the central $\mathrm{BH}$ increases and, correspondingly, the associated AGN feedback efficiency. In addition, AGN heating is supposed to be strong enough to reduce the star formation in the BCGs.

However, despite the strong reasons in favor of this AGN feedback cycle, implementing such a self-regulated mechanism in simulations represents a challenging task (e.g. Borgani \& Kravtsov 2011, and references therein). In this sense, the first attempts to build competent models of AGN heating consisted in theoretical studies accounting for the effects of AGN feedback out of cosmological context (e.g. Churazov et al. 2001; Quilis et al. 2001). In the last years, however, different implementations and refinements of AGN feedback models have been included in cosmological simulations (e.g. Springel et al. 2005; Sijacki et al. 2007, Puchwein et al. 2008, McCarthy et al. 2010, Puchwein et al. 2010; Fabjan et al. 2010, Short et al. 2010, Battaglia et al. 2013; Martizzi et al. 2012, Ragone-Figueroa et al. 2013). Given the limited spatial and temporal resolutions achievable by current simulations, phenomenological models are needed to include this form of energy feedback. In these models, the rates of AGN energy injection are usually computed by adopting the Bondi gas accretion onto the central SMBHs (Bondi 1952 11 In addition to thermal AGN feedback, some observations report that $\mathrm{BHs}$ in the center of galaxies generate relativistic jets that shock and heat the neighboring ICM. In the light of these observations, the effects of kinetic AGN feedback in the form of AGN-driven winds have been also analyzed by several authors (e.g. Omma et al. 2004; Dubois et al. 2011, Gaspari et al. 2011; Barai et al. 2014).

${ }^{11}$ It is important to point out that the Bondi approach is the simplest model of gas accretion. A number of studies (e.g. Hobbs et al. 2012) have already highlighted the main drawbacks of this approach and the necessity of adopting alternative and more realistic schemes. 

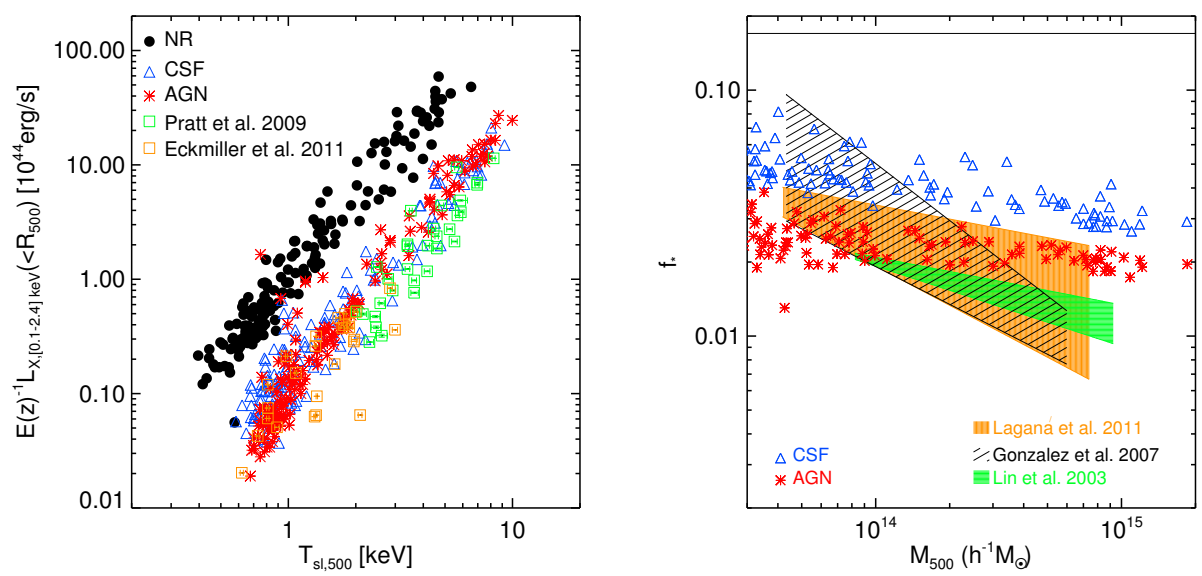

Fig. 15 Left panel: $L_{X}-T$ relation for the sample of groups and clusters identified in the simulations by Planelles et al. (2013b). Results are shown for a set of non-radiative simulations $(N R)$, and for two sets of radiative simulations, one including cooling, star formation, $\mathrm{SN}$ feedback and metal enrichment (CSF), and another one accounting as well for the effects of AGN feedback $(A G N)$. Figure from Planelles et al. (2013b). Right panel: Stellar mass fraction as a function of cluster mass as obtained in the simulations by Planelles et al. (2013a). Results from radiative simulations with $(A G N)$ and without (CSF) AGN feedback are shown. The horizontal continuous line stands for the assumed baryon mass fraction in the simulations. Figure from Planelles et al. (2013a). In both panels, data from different observational samples is used for comparison.

Simulations including different prescriptions of these phenomenological models have indeed reported some promising achievements (e.g. Sijacki et al. 2007, Puchwein et al. 2008, Fabjan et al. 2010; Martizzi et al. 2012, Planelles et al. 2013a b, Le Brun et al. 2013). As it is shown in the right panel of Fig. 15. AGN feedback seems to be very efficient in attenuating the star formation in high-mass galaxy clusters, producing therefore stellar mass fractions in better agreement with observational data. In addition, as shown in the left panel of Fig. 15. AGN heating can also reduce the amount of hot gas in small clusters and groups, thus reproducing better the observed $L_{X}-T$ relation and partially resolving the disagreement that otherwise existed for small systems (see top panel of Fig. 10). Besides, AGN feedback has been also shown to be quite effective in dispersing heavy elements throughout the intra-cluster plasma, producing a better consistency with the observed ICM metallicity profiles (e.g. Fabjan et al.|2010, McCarthy et al.|2010, Planelles et al.|2013b).

Despite the above successes, a number of discrepancies between observations and simulations still exist. As an example, Fig. 16 shows the mean temperature and entropy radial profiles for the sample of relaxed and unrelaxed massive clusters identified in the AGN simulations by Planelles et al. (2013b). The lack of diversity between the simulated profiles of relaxed and unrelaxed systems is at odds with the observed profiles of $\mathrm{CC}$ and NCC clusters. This indicates that, even including AGN feedback and accounting for metal-dependent cooling rates, simulations are still not able to produce the correct cooling/heating interplay in cluster cores. The entropy values in inner regions are also higher than reported by observations. In addition, although the stellar masses of the BCGs obtained in these simulations are reduced, they are still much larger than observed (Ragone-Figueroa et al. 2013, see as well Kravtsov et al. 2014 for a recent observational analysis of the stellar mass-halo mass relation). Moreover, in a recent work, Gaspari et al. (2014) investigated the isolated effect of kinetic and thermal AGN feedback on the $L_{X}-T$ relation of galaxy clusters and groups. 

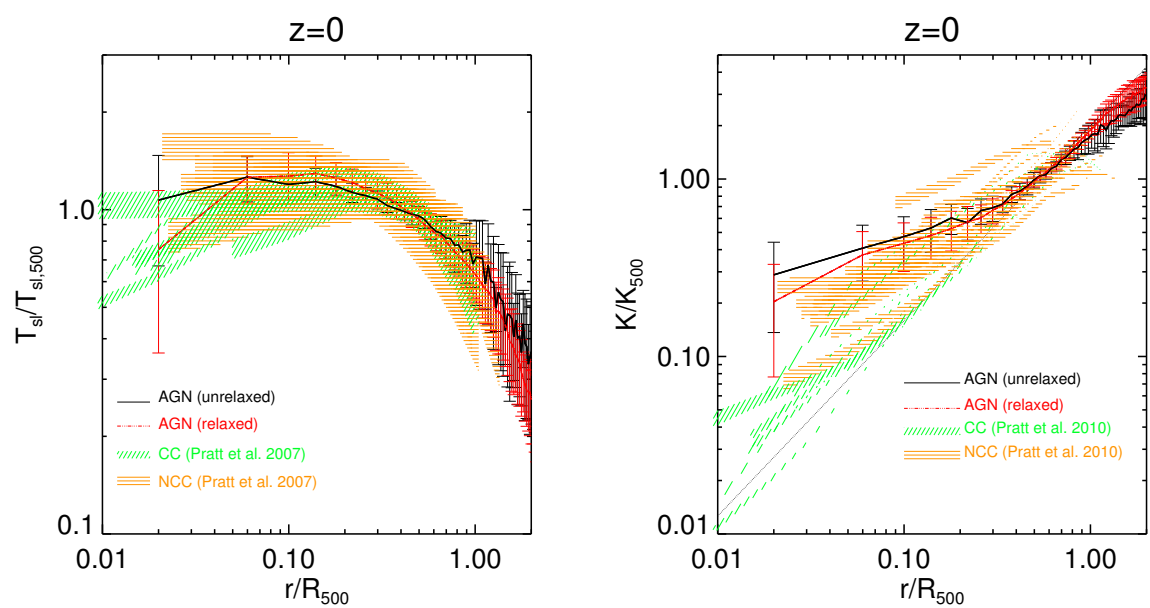

Fig. 16 Mean temperature (left panel) and entropy (right panel) radial profiles for the sample of relaxed/unrelaxed galaxy clusters identified in a set of simulations including AGN feedback (adapted from Planelles et al.|2013b). Error bars indicate $\pm 1-\sigma$ dispersion around the mean profile. In both panels, radial profiles of CC and NCC clusters from the REXCESS sample (Pratt et al. 2007 2010), which are represented by colored shadowy areas, are used for comparison. The dotted black line in the right panel shows the selfsimilar expectation for the entropy $\left(K \propto r^{1.1}\right)$.

They showed that, even with different parameterizations of these commonly used AGN models, it is not possible to break self-similarity to the desired level without actually breaking as well the cool-core structure of the considered systems.

These results suggest that, in order to describe the observational properties of the intracluster plasma in inner core regions and beyond, a proper scheme of AGN feedback may be complemented by additional physical processes. In this sense, a number of mechanisms, such as the effects of CRs in AGN-induced bubbles (e.g. Sijacki et al. 2008), the heating induced by galaxy motions (e.g. Kim et al.2005), or thermal conduction (e.g. Zakamska \& Narayan 2003), have been suggested. However, further investigation is required to find the correct interaction between these and additional plasma physical processes.

\subsection{Additional plasma physical processes}

Intergalactic gas heating at the cluster formation stage occurs by means of conversion of the energy of a cold gravitationally accelerated baryonic matter into the energy of hot thermal gas at cosmological shocks. The process of relaxation of the kinetic energy of the cold plasma flow to the quasi-equilibrium thermal distribution in rarefied cosmic plasmas is non-trivial. This is because the Coulomb collision rate is slow in the rarefied intergalactic medium and the relaxation processes are collisionless which means they are due to the collective plasma wave-particle interactions. Therefore, the standard textbook single fluid hydrodynamic and MHD approaches are not a priori valid for the description of the collisionless plasma flows. 
6.3.1 Gas heating, magnetic field amplification and particle acceleration in collisionless cosmological shocks

The Coulomb mean free path of a proton of velocity $v_{7}$ (measured in $100 \mathrm{~km} \mathrm{~s}^{-1}$ ) in the WHIM of overdensity $\delta$ can be estimated as $\lambda_{p} \approx 3.5 \times 10^{21} \cdot v_{7}^{4} \cdot \delta^{-1} \cdot(1+z)^{-3}$. $\left(\Omega_{b} h^{2} / 0.02\right)^{-1} \mathrm{~cm}$, where $\Omega_{b}$ is the baryon density parameter and we assume the Coulomb logarithm to be about 40 . The protons are magnetized in the flow (i.e. their gyro-frequencies are higher than the mean frequencies of the Coulomb collisions) if the magnetic field magnitude exceeds about $10^{-18} \mathrm{G}$.

The microscopic plasma scale, called the ion inertial length, is defined as $l_{i}=c / \omega_{\mathrm{pi}} \approx$ $2.3 \times 10^{7} n^{-0.5} \mathrm{~cm}$, where $\omega_{\mathrm{pi}}$ is the ion plasma frequency and $n$ is the ionized ambient gas number density measured in $\mathrm{cm}^{-3}$. This scale determines the widths of the transition region of the supercritical collisionless shock waves. In the strong enough collisionless shocks (typically of a Mach number above a few) resistivity cannot provide energy dissipation fast enough to create a standard shock transition on a microscopic scale (e.g. Kennel et al. 1985). Ion instabilities are important in such shocks, the so-called supercritical shocks. At the microscopic plasma scale the front of a supercritical shock wave is a transition region occupied by magnetic field fluctuations of an amplitude $\delta B / B \sim 1$ and characteristic frequencies of about the ion gyro-frequency. Generation of the fluctuations is due to instabilities in the interpenetrating multi-flow ion movements. The viscous transition region width is typically a few hundreds ion inertial lengths for a parallel shock, while it is about ten times shorter for a transverse shock. The ion inertial length in the WHIM can be estimated as $l_{i} \approx 5.1 \times 10^{10} \cdot \delta^{-1 / 2} \cdot(1+z)^{-3 / 2} \cdot\left(\Omega_{0} h^{2} / 0.02\right)^{-1 / 2} \mathrm{~cm}$, providing the width of the collisionless shock transition region is smaller by many orders of magnitude than the Coulomb mean free path (that is in the kiloparsec range). The question whether collisionless shocks can form in plasmas with magnetic pressure much smaller than the plasma pressure is of fundamental importance. Two-dimensional particle-in-cell (PIC) simulations of the structure of non-relativistic collisionless shocks in unmagnetized electron-ion plasmas performed by Kato \& Takabe (2008, 2010) revealed that the energy density of the magnetic field generated by the Weibel-type instability within the shock transition region reaches typically $1 \%-2 \%$ of the upstream bulk kinetic energy density. The width of the shock transition region was found in their simulation to be about 100 ion inertial lengths $l_{i}$, independent of the shock velocity. A tiny fraction (much less than a percent) of the incoming protons can be reflected from the collisionless shock transition region and these particles are subject of Fermi acceleration if the shock upstream flow carries magnetic fluctuations. In the case of strong shocks with high Alfvén and sonic Mach numbers the accelerated particles can get a substantial part (tens of percent) of the shock ram pressure. The pressure of non-thermal accelerated particles may mediate the shock flow as it was apparently observed by Voyager 2 in the heliosphere termination shock (see, e.g. Florinski et al. 2009). Moreover, evidences of strong non-adiabatic amplification of fluctuating magnetic fields by anisotropic distributions of accelerated particles observed in strong shocks of young supernova remnants (see, e.g. Helder et al. 2012).

Hybrid plasma simulations with kinetic treatment of ions and fluid electron description (see, e.g. Winske et al. 1990, Treumann 2009, Burgess \& Scholer 2013) allow us to study domains of some thousands gyroradii of incoming proton around the non-relativistic shocks. Recent two-dimensional hybrid simulations by Gargaté \& Spitkovsky (2012), who modelled quasi-parallel shock with the Alfvén Mach number $\mathscr{M}_{a}=6$ revealed energetic power-law ion distribution of index about -2 in the shock downstream. The energetic non-thermal particle population contained about $15 \%$ of the incoming upstream flow. Limited dynamical 


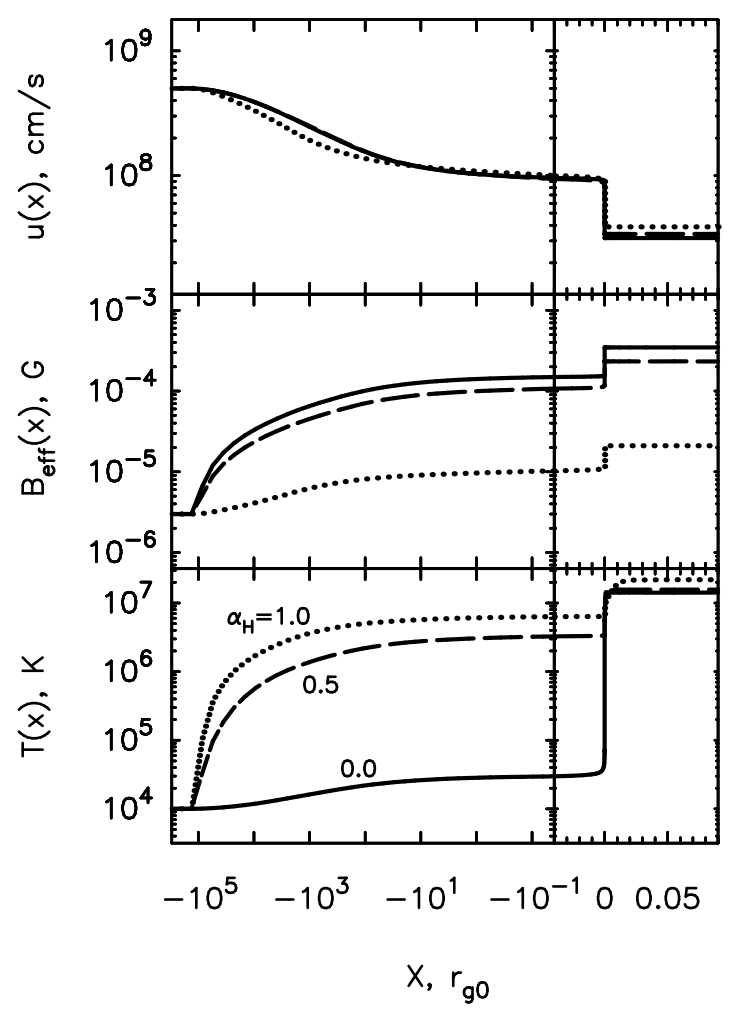

Fig. 17 Profile of a strong shock in WHIM simulated by Vladimirov et al. (2008) in the non-linear Monte Carlo model with different values of the fraction $\alpha_{H}$ of CR driven magnetic turbulence dissipated in the precursor. The solid, dashed and dotted lines correspond, respectively, to $\alpha_{H}=0,0.5$ and 1.0 . The plotted quantities are the bulk flow speed $u(x)$, the effective amplified magnetic field $B_{\text {eff }}(x)$ and the thermal gas temperature $T(x)$. The shock is located at $x=0$, and note the change from the logarithmic to the linear scale at $x=-0.05 r_{\mathrm{g} 0}$.

ranges of PIC and hybrid simulations do not allow us yet to study the formation of extended non-thermal tails of relativistic particles accelerated by non-relativistic shocks. On the other hand, simulations of DSA based on kinetic and Monte-Carlo modeling indicated the formation of the extended tails of the non-thermal particles. The energetic particles accelerated by a strong shock have hard spectral indexes and therefore, the CR pressure is dominated by the high-energy end of particle distribution. These particles may penetrate into far upstream and modify the shock flow by the CR-pressure gradient (see, e.g. Blandford \& Eichler 1987; Jones \& Ellison 1991, Malkov \& Drury 2001, Amato \& Blasi 2006, Vladimirov et al. 2008). Energetic particles of the highest energy escape into the upstream region providing energy outflow and allowing the shock compression ratio to be higher while the post-shock gas temperature and entropy appear significantly reduced comparing to that in the standard single fluid shock. It is important to note that the DSA is a very complicated highly nonequilibrium non-linear process with a strong coupling between the thermal and non-thermal components. Fast growing instabilities of the anisotropic distributions of energetic particles result in efficient production of strong magnetic turbulence in the shock upstream (see e.g. McKenzie \& Voelk 1982, Bell 2004; Bykov et al. 2012, Schure et al.,2012). 

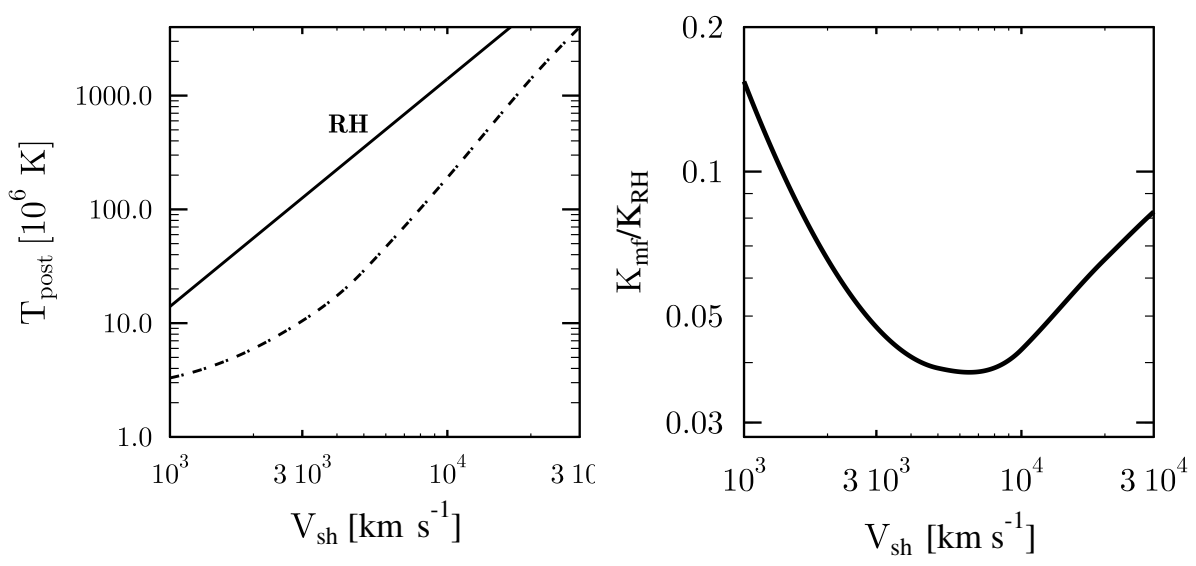

Fig. 18 Left panel: The post shock proton temperature as a function of shock velocity simulated by a nonlinear Monte Carlo model with an account of the efficient particle acceleration and magnetic field amplification. The far upstream gas temperature was about $2 \times 10^{4} \mathrm{~K}$ corresponding to the photo-ionized intergalactic gas accreting by a cluster. The postshock temperature (shown with dash-dotted line) was simulated for a model with turbulent cascade of CR-driven magnetic fluctuations Vladimirov et al. (2009). The solid curve (labeled as RH) is the standard Rankine-Hugoniot single fluid postshock temperature (it is presented for a comparison). Right panel: The ratio of the postshock gas entropy (labeled as $K_{\mathrm{mf}}$ multi-fluid) to the standard single fluid (Rankine-Hugoniot $K_{\mathrm{RH}}$ ) postshock gas entropy as a function of shock velocity simulated for cosmic ray modified collisionless shock.

In Fig. 17 we illustrate the effects mentioned above with simulated velocity, magnetic field, and gas temperature profiles of a strong shock of velocity $5000 \mathrm{~km} \mathrm{~s}^{-1}$ with the far upstream gas temperature of $10^{4} \mathrm{~K}$ and magnetic field of about $\mu \mathrm{G}$. The simulation was made with the non-linear Monte-Carlo model by Vladimirov et al. (2008, 2009) which accounts for efficient $\mathrm{CR}$ acceleration, strong non-adiabatic magnetic field amplification due to CR-driven instabilities in the shock upstream with magnetic turbulence dissipation, and the escape of highest energy particles to the shock upstream. The models of non-linear DSA predict hard spectra of accelerated relativistic particles, which often show concave spectral shapes instead of the power-laws. Strong amplification of the fluctuating magnetic fields in the upstream flow by CR-driven instabilities is expected in the models of DSA. Important physical effects to be learned from the strong collisionless shock modeling are: (i) potentially sizeable (above ten percent) energy leakage from the system with the ultra-relativistic particles accelerated at the shock and escaping through the shock upstream back into intergalactic medium, and (ii) a possibility of strong super-adiabatic magnetic field amplification by CR-driven instabilities (see for a review Bykov et al. 2013).

These effects may strongly affect the thermal properties of shocks with high sonic and alfvenic Mach numbers as it is expected to be the case in the external accreting shocks at cluster outskirts. In Fig. 18 we illustrate the possible effect of the non-thermal components on the ion temperature (left panel) and the entropy (right panel) in the downstream of the multi-fluid shock simulated with non-linear Monte-Carlo model described in Vladimirov et al. (2009). Both post-shock ion temperature and gas entropy $K_{\mathrm{mf}}$ in the multi-fluid collisionless shock can be strongly reduced compared to that in the standard single fluid RankineHugoniot adiabat. This is because of a substantial increase of the gas compression ratio in a strong collisionless shock converting a sizable part of the shock ram pressure into relativistic 
particles. Some fraction of the accelerated particles escape the system thus allowing the gas compression ratio to be much larger than 4 . As it was discussed in $\S 6.1$ most of the kinetic energy dissipation occurs at the cluster inner shocks with the modest Mach numbers where the effects discussed above are likely much less prominent. Indeed, the ratio of the thermal gas heating to CR acceleration rates in weak shocks of sonic Mach number $\mathscr{M}_{\mathrm{s}}<2$ is proportional to $\left(\mathscr{M}_{\mathrm{s}}-1\right) \beta_{\mathrm{p}}$, providing inefficient $\mathrm{CR}$ acceleration by weak shocks in the case of the large ratio of the thermal and magnetic pressures $\beta_{\mathrm{p}} \gg 1$ expected in the inner regions of the cluster. A recent search of $\gamma$-ray emission from stacked Fermi-LAT count maps of some dozens of clusters of galaxies (The Fermi-LAT Collaboration et al. 2013, Dutson et al.2013; Huber et al. 2013) established stringent upper limits on the average CR to thermal pressure ratio to be below of a few percent within the radius $r_{200}$ depending on the assumed index of the power-law CR distribution and $\gamma$-ray background models. Detection of $\gamma$-ray emission from extended regions around the external accretion shocks in the vicinity of filaments and clusters is a challenging task given its low surface brightness because of the low gas density.

\section{Concluding remarks and open problems}

In this review, we have discussed recent results on structure formation focusing our attention on the first objects in the Universe and the most massive clusters of galaxies at the present day. These extreme scenarios allow us to clearly illustrate the relevance of the physics of plasma on the formation of cosmic structures along a wide range of spatial and temporal scales. In the hierarchical paradigm of structure formation, the first objects are the building blocks of subsequent structure formation, leading to larger galaxies and galaxy clusters through accretion and merger events (e.g. Somerville et al. 2012). Despite the disparity of involved scales, a number of physical processes, such as radiative cooling, turbulence and feedback, appear to be common, suggesting them to be ubiquitous in the non-linear regime of cosmic structure formation.

In the early Universe, the first objects are expected to form in halos with $10^{5}-10^{8} \mathrm{M}_{\odot}$ at redshift 10 - 30 (e.g. Bromm et al. 2009, Bromm \& Yoshida 2011). Here we distinguish the so-called minihalos with virial temperatures above $1000 \mathrm{~K}$ from the atomic cooling halos with virial temperatures above $10^{4} \mathrm{~K}$. Minihalos are the expected formation sites for the first primordial stars, with typical masses in the range of $10-100 \mathrm{M}_{\odot}$ (Abel et al. 2002; Bromm et al. 2002, Yoshida et al. 2008, Clark et al. 2011, Greif et al. 2011, Hosokawa et al. 2011, Turk et al. 2012; Latif et al. 2013c; Susa 2013). Their formation is governed by the chemistry and cooling of molecular hydrogen, as well as additional processes such as turbulence (e.g. Turk et al. 2012; Latif et al. 2013c), radiative feedback (e.g. Hosokawa et al. 2011, Susa 2013) and magnetic fields (e.g. Tan \& Blackman 2004, Machida et al. 2006; Sur et al. 2010; Schober et al. 2012, Sur et al. 2012, Turk et al.|2012).

The atomic cooling halos show a more complex evolution depending on their local conditions, in particular regarding their metallicity and dust content. In this review, we restricted ourselves to the formation of massive black holes in primordial halos (see Fig. 19 for an illustrative summary), while a more general discussion is given by Bromm \& Yoshida (2011). In the presence of strong photodissociating backgrounds, $\mathrm{H}_{2}$ formation is suppressed (Omukai) 2001, Machacek et al. 2001; Johnson et al. 2008, Schleicher et al.|2010b; Shang et al. 2010, Latif et al. 2011), leading to a close-to-isothermal collapse regulated via atomic hydrogen lines. Recent numerical simulations confirm that massive central objects can indeed form, due to the high accretion rates of more than $1 \mathrm{M}_{\odot} \mathrm{yr}^{-1}$ (Latif et al. 2013a). In the presence of such accretion rates, feedback can be expected to be weak (Hosokawa et al. 2012, Schle- 


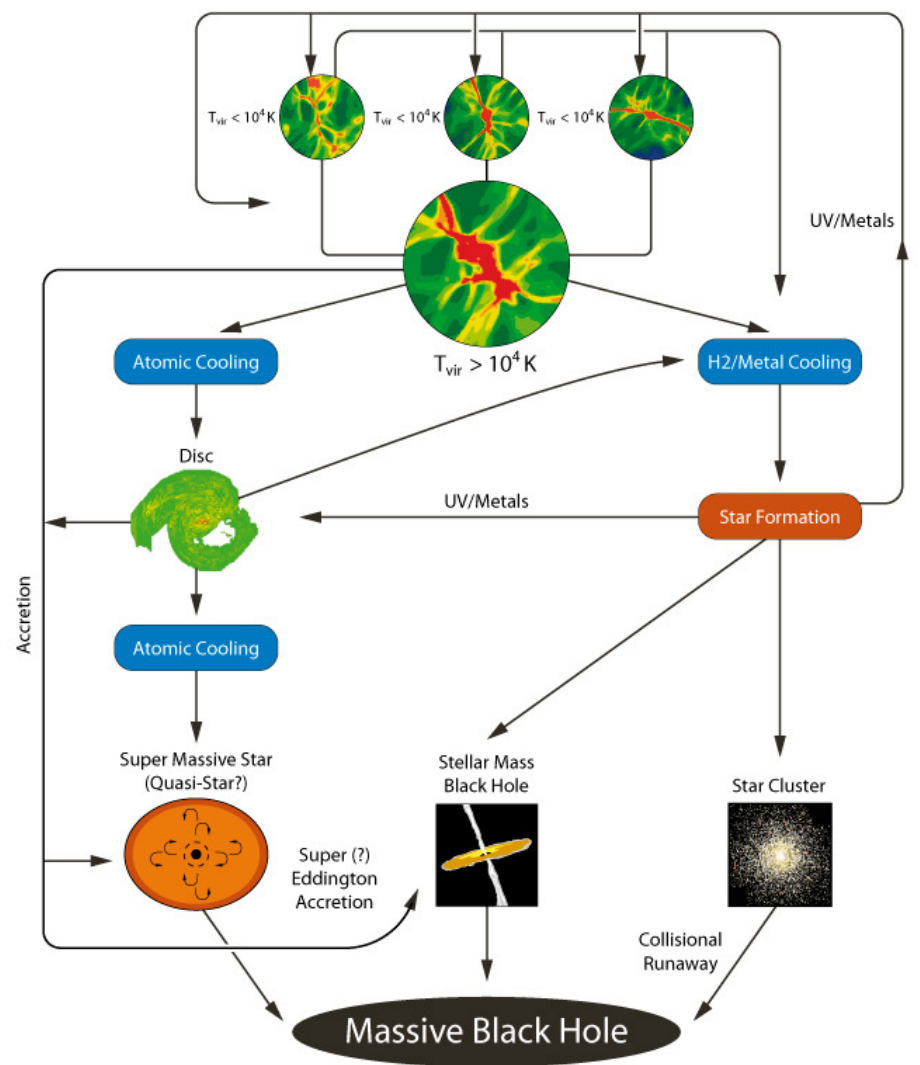

Fig. 19 Flowchart summarizing possible paths for the formation of the first SMBHs in high-redshift atomic cooling halos. Figure from Regan \& Haehnelt (2009a).

icher et al. 2013) and does not impede the accretion. Indeed, even trace amounts of dust, corresponding to $10^{-5}-10^{-3}$ times the dust-to-gas ratio in the solar neighborhood, may already trigger strong cooling and fragmentation at high densities (Schneider et al. 2004, Omukai et al. 2005), but also stimulate the formation of molecular hydrogen at low to moderate densities (Cazaux \& Spaans 2009, Latif et al. 2012). The extremely metal poor star SDSS J1029151+172927 (Caffau et al. 2011) shows chemical abundances at which metal line coolant is inefficient, and where only trace amounts of dust grains were able to trigger cooling and fragmentation (Klessen et al. 2012; Schneider et al. 2012, ${ }^{12}$. For metallicities above $10^{-2}$ solar, on the other hand, metal line cooling can be expected to be significant (Bromm \& Loeb 2003b; Omukai et al. 2005). The fragmentation of such metal-enriched atomic cooling halos is in fact poorly understood (see e.g. Safranek-Shrader et al. 2010, for first modeling attempts) and needs to be investigated in further detail.

${ }^{12}$ An alternative formation scenario for the star SDSS J1029151+172927 has been recently proposed by MacDonald et al. (2013), who suggest that it may have been a subgiant formed with significantly higher metallicity in the vicinity of a SN-Ia. 
Cluster observables: Physical processes in clusters:

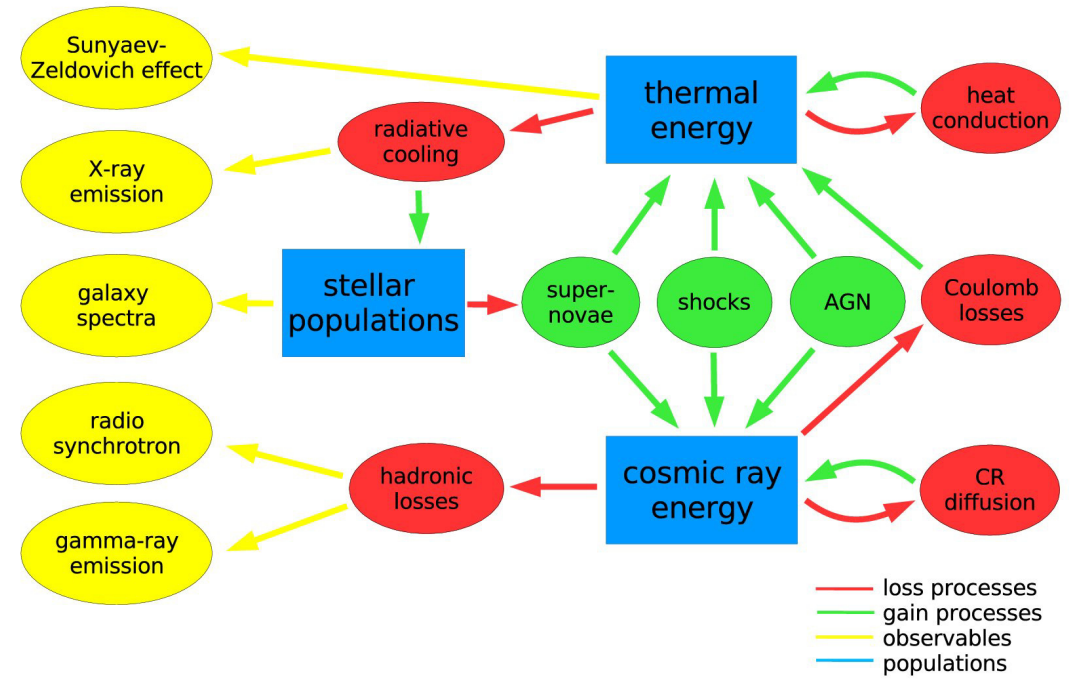

Fig. 20 Flowchart summarizing the connections between the main physical processes taking place in galaxy clusters together with the different observational channels through which they can be detected. Figure from Pfrommer et al. (2008).

In the hierarchical paradigm of structure formation, where the first objects are the building blocks of subsequent structure development, clusters of galaxies, with masses of up to $10^{15} M_{\odot}$ at $z=0$, occupy the most massive extreme of the cosmic hierarchy. The formation and evolution of galaxy clusters is a complex and non-linear event resulting from the intricate interaction of a number of physical processes acting on a wide range of scales (see Kravtsov \& Borgani 2012, for a recent review and references therein). As an example, Fig. 20 shows a simplified summary of some of the main processes operating in galaxy clusters. On large scales, the hierarchical process of structure formation induces the development of strong cosmological shocks, surrounding galaxy clusters and filaments, that contribute to heat and compress the hot intra-cluster plasma. Within galaxy clusters, weaker internal shocks, mainly originated by subhalo mergers or accretion phenomena, change the energetic balance of the gas and allow the halos to virialize. These shocks can also generate ICM turbulence and mixing, amplify magnetic fields, and accelerate thermal distributions of particles giving rise to a non-thermal population of CRs. In dense regions within galaxies and galaxy clusters, the intra-cluster gas can cool radiatively, leading to both star formation and gas accretion onto SMBHs residing at the center of massive cluster galaxies. These processes can then provide a significant energy contribution to the ICM in the form of SNe explosions or AGN feedback. As shown in Fig. 20, all these processes, which are highly interconnected between them, are manifested by means of different observational channels.

In the last years, the new generations of supercomputing and programming facilities have been crucial to deepen in our understanding of the complicated physical processes taking place within the intra-cluster plasma and shaping the observational properties of galaxy clusters. In order to explain the observations, cosmological hydrodynamical simulations have tried to implement the most relevant physical processes self-consistently with 
the cosmic evolution. In particular, in addition to gravitationally-induced phenomena inherent to structure formation, the standard non-gravitational processes commonly included in these simulations are radiative cooling, star formation and SN feedback. In the last years, the inclusion of the effects of thermal and/or kinetic AGN feedback is also becoming a common practice, despite the fact that the particularities of the heating mechanism are still uncertain. In spite of the relatively simplicity employed in modeling these complex processes, simulations have been able to significantly reproduce most of the observational cluster properties, at least for massive systems at relatively outer cluster regions $\left(0.1 R_{500} \lesssim r \lesssim R_{500}\right)$, where clusters are assumed to be nearly self-similar. However, inner cluster regions and smaller systems show a number of significant issues that still need to be solved. In these inner regions, simulations still show an excess of gas cooling, which produces an excess in both the star formation and the metal production. In addition, simulations are still not able to solve the cooling flow problem or to reproduce the diversity of the observed temperature and entropy radial profiles of relaxed and unrelaxed systems. On the other hand, cluster outskirts $\left(r \gtrsim R_{500}\right)$ are also affected by strong deviations from hydrostatic equilibrium caused, primarily, by sources of non-thermal pressure support such as CRs or magnetic fields, which generally are not modeled in simulations. These results indicate that, in addition to the processes already included, a number of additional physical processes, mainly related with the complex physics of plasma, such as turbulence, viscosity or thermal conduction, must be also properly taken into account. Therefore, although AGN feedback seems to be the most favored energy source to regulate cooling in clusters, a subtle interplay with a number of supplementary physical phenomena may be needed to explain the observational properties of galaxy clusters and groups, from the core regions out to the outskirts.

In the near future, a significant numerical effort will be aimed at performing larger and better-resolved cosmological simulations with a more accurate modeling of the physics of galaxy evolution. In addition to these technical improvements, forthcoming instruments, like the JWST (Gardner et al.2006) and the new generation of large ground-based telescopes, are expected to detect light from the first galaxies, contributing to interpret early structure formation. Besides, a number of large observational surveys in different wavebands, such as eROSITA (Pillepich et al. 2012), Euclid (Laureijs et al. 2012), WFXT (Pareschi \& Campana 2011) or the LSST (LSST Science Collaboration et al. 2009), will provide a significantly large number of clusters. These numerical and observational efforts, together with a more accurate treatment of the physics of plasma, will definitely shed some more light on the nature of the physical processes governing the formation of structures in the Universe, from the first non-linear objects to the present-day massive galaxy clusters.

Acknowledgements We would like to thank the ISSI staff for their hospitality and for providing an inspiring atmosphere at the International Space Science Institute Workshop in Bern in 2013. We also would like to thank the anonymous referee for his/her constructive comments. SP acknowledges support by the PRININAF09 project "Towards an Italian Network for Computational Cosmology" and by the PRIN-MIUR09 "Tracing the growth of structures in the Universe". DRGS thanks for funding from the German Science Foundation (DFG) in the DFG priority program SPP 1573 Physics of the Interstellar Medium under grant SCHL 1964/1-1, and via the collaborative research center (CRC) 963/1 Astrophysical flow instabilities and turbulence (project A12). AMB was supported in part by RAS Presidium and OFN 15 and 17 programs. We further thank for stimulating discussions with Stefano Borgani, Stefano Bovino, Muhammad Latif, Wolfram Schmidt, Jens Niemeyer and Barbara Sartoris.

\section{References}

Abel, T., Anninos, P., Zhang, Y., \& Norman, M. L. 1997, New Astronomy, 2, 181 
Abel, T., Bryan, G. L., \& Norman, M. L. 2002, Science, 295, 93

Agarwal, B., Khochfar, S., Johnson, J. L., et al. 2012, MNRAS, 425, 2854

Agertz, O., Moore, B., Stadel, J., et al. 2007, MNRAS, 380, 963

Allen, S. W., Evrard, A. E., \& Mantz, A. B. 2011, ARAA, 49, 409

Allen, S. W., Schmidt, R. W., \& Fabian, A. C. 2001, MNRAS, 328, L37

Amato, E. \& Blasi, P. 2006, MNRAS, 371, 1251

Angulo, R. E., Springel, V., White, S. D. M., et al. 2012, MNRAS, 426, 2046

Arnaud, M. \& Evrard, A. E. 1999, MNRAS, 305, 631

Arnaud, M., Pointecouteau, E., \& Pratt, G. W. 2005, A\&A, 441, 893

Audit, E., Teyssier, R., \& Alimi, J.-M. 1997, A\&A, 325, 439

Ball, R., Burns, J. O., \& Loken, C. 1993, AJ, 105, 53

Balogh, M. L., Babul, A., \& Patton, D. R. 1999, MNRAS, 307, 463

Balogh, M. L., Pearce, F. R., Bower, R. G., \& Kay, S. T. 2001, MNRAS, 326, 1228

Barai, P., Viel, M., Murante, G., Gaspari, M., \& Borgani, S. 2014, MNRAS, 437, 1456

Barkana, R. \& Loeb, A. 2001, Phys. Rep., 349, 125

Battaglia, N., Bond, J. R., Pfrommer, C., \& Sievers, J. L. 2013, ApJ, 777, 123

Begelman, M. C., Volonteri, M., \& Rees, M. J. 2006, MNRAS, 370, 289

Bell, A. R. 2004, MNRAS, 353, 550

Bertschinger, E. 1985, ApJS, 58, 39

Bhattacharya, S., Heitmann, K., White, M., et al. 2011, ApJ, 732, 122

Binney, J. \& Tremaine, S. 2008, Galactic Dynamics: Second Edition (Princeton University Press)

Bîrzan, L., Rafferty, D. A., McNamara, B. R., Wise, M. W., \& Nulsen, P. E. J. 2004, ApJ, 607, 800

Blandford, R. \& Eichler, D. 1987, Phys. Rep., 154, 1

Blumenthal, G. R., Faber, S. M., Primack, J. R., \& Rees, M. J. 1984, Nature, 311, 517

Bonafede, A., Giovannini, G., Feretti, L., Govoni, F., \& Murgia, M. 2009, A\&A, 494, 429

Bond, J. R., Cole, S., Efstathiou, G., \& Kaiser, N. 1991, ApJ, 379, 440

Bondi, H. 1952, MNRAS, 112, 195

Borgani, S. 2008, in Lecture Notes in Physics, Berlin Springer Verlag, Vol. 740, A PanChromatic View of Clusters of Galaxies and the Large-Scale Structure, ed. M. Plionis, O. López-Cruz, \& D. Hughes, 287

Borgani, S., Diaferio, A., Dolag, K., \& Schindler, S. 2008, Sp. Sc. Rev., 134, 269

Borgani, S., Governato, F., Wadsley, J., et al. 2001, ApJ, 559, L71

Borgani, S. \& Guzzo, L. 2001, Nature, 409, 39

Borgani, S. \& Kravtsov, A. 2011, Advanced Science Letters, 4, 204

Borgani, S., Murante, G., Springel, V., et al. 2004, MNRAS, 348, 1078

Bournaud, F., Elmegreen, B. G., Teyssier, R., Block, D. L., \& Puerari, I. 2010, MNRAS, 409, 1088

Bovino, S., Schleicher, D. R. G., \& Grassi, T. 2014, A\&A, 561, A13

Braun, H. \& Schmidt, W. 2012, MNRAS, 421, 1838

Bromm, V., Coppi, P. S., \& Larson, R. B. 2002, ApJ, 564, 23

Bromm, V. \& Larson, R. B. 2004, ARAA, 42, 79

Bromm, V. \& Loeb, A. 2003a, ApJ, 596, 34

Bromm, V. \& Loeb, A. 2003b, Nature, 425, 812

Bromm, V. \& Yoshida, N. 2011, ARAA, 49, 373

Bromm, V., Yoshida, N., Hernquist, L., \& McKee, C. F. 2009, Nature, 459, 49

Bryan, G. L. \& Norman, M. L. 1998, ApJ, 495, 80

Bryan, G. L., Norman, M. L., O’Shea, B. W., et al. 2014, ApJS, 211, 19 
Burgess, D. \& Scholer, M. 2013, Sp. Sc. Rev., 178, 513

Burns, J. O. 1990, AJ, 99, 14

Bykov, A. M., Bloemen, H., \& Uvarov, Y. A. 2000, A\&A, 362, 886

Bykov, A. M., Brandenburg, A., Malkov, M. A., \& Osipov, S. M. 2013, Sp. Sc. Rev., 178, 201

Bykov, A. M., Dolag, K., \& Durret, F. 2008, Sp. Sc. Rev., 134, 119

Bykov, A. M., Ellison, D. C., \& Renaud, M. 2012, Sp. Sc. Rev., 166, 71

Caffau, E., Bonifacio, P., François, P., et al. 2011, Nature, 477, 67

Cavagnolo, K. W., Donahue, M., Voit, G. M., \& Sun, M. 2009, ApJS, 182, 12

Cazaux, S. \& Spaans, M. 2009, A\&A, 496, 365

Cen, R. \& Ostriker, J. P. 1999, ApJ, 514, 1

Chandran, B. D. G., Sharma, P., \& Parrish, I. J. 2009, in Astronomy, Vol. 2010, astro2010: The Astronomy and Astrophysics Decadal Survey, 41

Chen, Y., Reiprich, T. H., Böhringer, H., Ikebe, Y., \& Zhang, Y.-Y. 2007, A\&A, 466, 805

Churazov, E., Brüggen, M., Kaiser, C. R., Böhringer, H., \& Forman, W. 2001, ApJ, 554, 261

Clark, P. C., Glover, S. C. O., Smith, R. J., et al. 2011, Science, 331, 1040

Cohn, J. D. \& White, M. 2008, MNRAS, 385, 2025

Coles, P. \& Lucchin, F. 2002, Cosmology: The Origin and Evolution of Cosmic Structure, Second Edition

Coppola, C. M., D’Introno, R., Galli, D., Tennyson, J., \& Longo, S. 2012, ApJS, 199, 16

Courtin, J., Rasera, Y., Alimi, J.-M., et al. 2011, MNRAS, 410, 1911

Crocce, M., Fosalba, P., Castander, F. J., \& Gaztañaga, E. 2010, MNRAS, 403, 1353

Cui, W., Borgani, S., Dolag, K., Murante, G., \& Tornatore, L. 2012, MNRAS, 423, 2279

Cui, W., Borgani, S., \& Murante, G. 2014, ArXiv e-prints

Cusworth, S. J., Kay, S. T., Battye, R. A., \& Thomas, P. A. 2014, MNRAS, 439, 2485

Dai, X., Bregman, J. N., Kochanek, C. S., \& Rasia, E. 2010, ApJ, 719, 119

Davé, R., Cen, R., Ostriker, J. P., et al. 2001, ApJ, 552, 473

Davé, R., Katz, N., \& Weinberg, D. H. 2002, ApJ, 579, 23

Davis, M., Efstathiou, G., Frenk, C. S., \& White, S. D. M. 1985, ApJ, 292, 371

Davis, M. \& Peebles, P. J. E. 1983, ApJ, 267, 465

De Grandi, S. \& Molendi, S. 2002, ApJ, 567, 163

Diemand, J., Kuhlen, M., \& Madau, P. 2007, ApJ, 667, 859

Dijkstra, M., Haiman, Z., Mesinger, A., \& Wyithe, J. S. B. 2008, MNRAS, 391, 1961

Dobbs, C. L., Theis, C., Pringle, J. E., \& Bate, M. R. 2010, MNRAS, 403, 625

Dolag, K., Bartelmann, M., \& Lesch, H. 1999, A\&A, 348, 351

Dolag, K., Borgani, S., Schindler, S., Diaferio, A., \& Bykov, A. M. 2008, Sp. Sc. Rev., 134, 229

Dolag, K., Vazza, F., Brunetti, G., \& Tormen, G. 2005, MNRAS, 364, 753

Drury, L. O. \& Falle, S. A. E. G. 1986, MNRAS, 223, 353

Dubois, Y., Devriendt, J., Teyssier, R., \& Slyz, A. 2011, MNRAS, 417, 1853

Dutson, K. L., White, R. J., Edge, A. C., Hinton, J. A., \& Hogan, M. T. 2013, MNRAS, 429, 2069

Eckert, D., Molendi, S., Vazza, F., Ettori, S., \& Paltani, S. 2013, A\&A, 551, A22

Edge, A. C. \& Frayer, D. T. 2003, ApJ, 594, L13

Eilek, J. A. 2004, in The Riddle of Cooling Flows in Galaxies and Clusters of galaxies, ed. T. Reiprich, J. Kempner, \& N. Soker, 165

Eisenstein, D. J. \& Hu, W. 1999, ApJ, 511, 5

Eke, V. R., Navarro, J. F., \& Frenk, C. S. 1998, ApJ, 503, 569 
Ensslin, T. A., Biermann, P. L., Klein, U., \& Kohle, S. 1998, A\&A, 332, 395

Ettori, S., Donnarumma, A., Pointecouteau, E., et al. 2013, Sp. Sc. Rev., 177, 119

Ettori, S., Tozzi, P., Borgani, S., \& Rosati, P. 2004, A\&A, 417, 13

Evrard, A. E. \& Henry, J. P. 1991, ApJ, 383, 95

Evrard, A. E., MacFarland, T. J., Couchman, H. M. P., et al. 2002, ApJ, 573, 7

Fabian, A. C., Crawford, C. S., Edge, A. C., \& Mushotzky, R. F. 1994, MNRAS, 267, 779

Fabjan, D., Borgani, S., Tornatore, L., et al. 2010, MNRAS, 401, 1670

Fabjan, D., Tornatore, L., Borgani, S., Saro, A., \& Dolag, K. 2008, MNRAS, 386, 1265

Fan, X., Narayanan, V. K., Lupton, R. H., et al. 2001, AJ, 122, 2833

Fan, X., Strauss, M. A., Schneider, D. P., et al. 2003, AJ, 125, 1649

Federrath, C., Sur, S., Schleicher, D. R. G., Banerjee, R., \& Klessen, R. S. 2011, ApJ, 731, 62

Ferrarese, L. \& Merritt, D. 2000, ApJ, 539, L9

Ferrari, C., Govoni, F., Schindler, S., Bykov, A. M., \& Rephaeli, Y. 2008, Sp. Sc. Rev., 134, 93

Florinski, V., Decker, R. B., le Roux, J. A., \& Zank, G. P. 2009, Geophys. Res. Lett., 36, 12101

Flower, D. R. \& Harris, G. J. 2007, MNRAS, 377, 705

Forrey, R. C. 2013, ApJ, 773, L25

Galli, D. \& Palla, F. 1998, A\&A, 335, 403

Gardner, J. P., Mather, J. C., Clampin, M., et al. 2006, Sp. Sc. Rev., 123, 485

Gargaté, L. \& Spitkovsky, A. 2012, ApJ, 744, 67

Gaspari, M., Brighenti, F., Temi, P., \& Ettori, S. 2014, ApJ, 783, L10

Gaspari, M., Melioli, C., Brighenti, F., \& D'Ercole, A. 2011, MNRAS, 411, 349

Giodini, S., Lovisari, L., Pointecouteau, E., et al. 2013, Sp. Sc. Rev., 177, 247

Giodini, S., Pierini, D., Finoguenov, A., et al. 2009, ApJ, 703, 982

Giovannini, G., Bonafede, A., Feretti, L., et al. 2009, A\&A, 507, 1257

Glover, S. 2005, Sp. Sc. Rev., 117, 445

Glover, S. C. O., Clark, P. C., Greif, T. H., et al. 2008, in IAU Symposium, Vol. 255, IAU Symposium, ed. L. K. Hunt, S. C. Madden, \& R. Schneider, 3-17

Gonzalez, A. H., Zaritsky, D., \& Zabludoff, A. I. 2007, ApJ, 666, 147

Grassi, T., Bovino, S., Schleicher, D. R. G., et al. 2014, MNRAS, 439, 2386

Greif, T. H., Bromm, V., Clark, P. C., et al. 2012, MNRAS, 424, 399

Greif, T. H., Glover, S. C. O., Bromm, V., \& Klessen, R. S. 2010, ApJ, 716, 510

Greif, T. H., Springel, V., White, S. D. M., et al. 2011, ApJ, 737, 75

Gunn, J. E. \& Gott, III, J. R. 1972, ApJ, 176, 1

Guth, A. H. \& Pi, S.-Y. 1982, Physical Review Letters, 49, 1110

Hamilton, J.-C. 2013, ArXiv e-prints

Häring, N. \& Rix, H.-W. 2004, ApJ, 604, L89

Helder, E. A., Vink, J., Bykov, A. M., et al. 2012, Sp. Sc. Rev., 173, 369

Helsdon, S. F. \& Ponman, T. J. 2000, MNRAS, 315, 356

Hobbs, A., Power, C., Nayakshin, S., \& King, A. R. 2012, MNRAS, 421, 3443

Hosokawa, T., Omukai, K., \& Yorke, H. W. 2012, ApJ, 756, 93

Hosokawa, T., Omukai, K., Yoshida, N., \& Yorke, H. W. 2011, Science, 334, 1250

Huber, B., Tchernin, C., Eckert, D., et al. 2013, A\&A, 560, A64

Iapichino, L. \& Niemeyer, J. C. 2008, MNRAS, 388, 1089

Jeans, J. H. 1902, Royal Society of London Philosophical Transactions Series A, 199, 1

Jenkins, A., Frenk, C. S., White, S. D. M., et al. 2001, MNRAS, 321, 372

Johnson, J. L., Dalla, V. C., \& Khochfar, S. 2013a, MNRAS, 428, 1857 
Johnson, J. L., Greif, T. H., \& Bromm, V. 2007, ApJ, 665, 85

Johnson, J. L., Greif, T. H., \& Bromm, V. 2008, MNRAS, 388, 26

Johnson, J. L., Whalen, D. J., Li, H., \& Holz, D. E. 2013b, ApJ, 771, 116

Jones, F. C. \& Ellison, D. C. 1991, Space Science Reviews, 58, 259

Kaiser, N. 1986, MNRAS, 222, 323

Kang, H. \& Jones, T. W. 2005, ApJ, 620, 44

Kang, H., Jones, T. W., \& Gieseler, U. D. J. 2002, ApJ, 579, 337

Kang, H., Ryu, D., Cen, R., \& Ostriker, J. P. 2007, ApJ, 669, 729

Kato, T. N. \& Takabe, H. 2008, ApJ, 681, L93

Kato, T. N. \& Takabe, H. 2010, ApJ, 721, 828

Kay, S. T., Thomas, P. A., Jenkins, A., \& Pearce, F. R. 2004, MNRAS, 355, 1091

Kennel, C. F., Edmiston, J. P., \& Hada, T. 1985, A quarter century of collisionless shock research, American Geophysical Union Geophysical Monograph Series, 34, 1

Kettula, K., Finoguenov, A., Massey, R., et al. 2013, ApJ, 778, 74

Kim, W.-T., El-Zant, A. A., \& Kamionkowski, M. 2005, ApJ, 632, 157

Klessen, R. S., Glover, S. C. O., \& Clark, P. C. 2012, MNRAS, 421, 3217

Klypin, A. A. \& Shandarin, S. F. 1983, MNRAS, 204, 891

Knebe, A., Knollmann, S. R., Muldrew, S. I., et al. 2011, MNRAS, 415, 2293

Knebe, A., Pearce, F. R., Lux, H., et al. 2013, MNRAS, 435, 1618

Koushiappas, S. M., Bullock, J. S., \& Dekel, A. 2004, MNRAS, 354, 292

Kravtsov, A., Vikhlinin, A., \& Meshscheryakov, A. 2014, ArXiv e-prints

Kravtsov, A. V. \& Borgani, S. 2012, ARAA, 50, 353

Lacey, C. \& Cole, S. 1993, MNRAS, 262, 627

Lacey, C. \& Cole, S. 1994, MNRAS, 271, 676

Laganá, T. F., Zhang, Y.-Y., Reiprich, T. H., \& Schneider, P. 2011, ApJ, 743, 13

Latif, M. A., Schleicher, D. R. G., Schmidt, W., \& Niemeyer, J. 2013a, MNRAS, 433, 1607

Latif, M. A., Schleicher, D. R. G., Schmidt, W., \& Niemeyer, J. 2013b, MNRAS, 430, 588

Latif, M. A., Schleicher, D. R. G., Schmidt, W., \& Niemeyer, J. 2013c, ApJ, 772, L3

Latif, M. A., Schleicher, D. R. G., Schmidt, W., \& Niemeyer, J. 2013d, MNRAS, 432, 668

Latif, M. A., Schleicher, D. R. G., \& Spaans, M. 2012, A\&A, 540, A101

Latif, M. A., Schleicher, D. R. G., Spaans, M., \& Zaroubi, S. 2011, A\&A, 532, A66

Laureijs, R., Gondoin, P., Duvet, L., et al. 2012, in Society of Photo-Optical Instrumentation

Engineers (SPIE) Conference Series, Vol. 8442

Le Brun, A. M. C., McCarthy, I. G., Schaye, J., \& Ponman, T. J. 2013, ArXiv e-prints

Leccardi, A. \& Molendi, S. 2008a, A\&A, 487, 461

Leccardi, A. \& Molendi, S. 2008b, A\&A, 486, 359

Lin, Y.-T., Mohr, J. J., \& Stanford, S. A. 2003, ApJ, 591, 749

Lodato, G. \& Natarajan, P. 2007, MNRAS, 377, L64

LSST Science Collaboration, Abell, P. A., Allison, J., et al. 2009, ArXiv e-prints

Lukić, Z., Heitmann, K., Habib, S., Bashinsky, S., \& Ricker, P. M. 2007, ApJ, 671, 1160

MacDonald, J., Lawlor, T. M., Anilmis, N., \& Rufo, N. F. 2013, MNRAS, 431, 1425

Machacek, M. E., Bryan, G. L., \& Abel, T. 2001, ApJ, 548, 509

Machida, M. N., Omukai, K., Matsumoto, T., \& Inutsuka, S.-i. 2006, ApJ, 647, L1

Maggiore, M. \& Riotto, A. 2010, ApJ, 711, 907

Magorrian, J., Tremaine, S., Richstone, D., et al. 1998, AJ, 115, 2285

Maier, A., Iapichino, L., Schmidt, W., \& Niemeyer, J. C. 2009, ApJ, 707, 40

Malkov, M. A. \& Drury, L. 2001, Reports on Progress in Physics, 64, 429

Markevitch, M. 1998, ApJ, 504, 27

Markevitch, M. \& Vikhlinin, A. 2007, Phys. Rep., 443, 1 
Martizzi, D., Teyssier, R., \& Moore, B. 2012, MNRAS, 420, 2859

Maughan, B. J., Giles, P. A., Randall, S. W., Jones, C., \& Forman, W. R. 2012, MNRAS, 421, 1583

Mayer, L., Governato, F., \& Kaufmann, T. 2008, Advanced Science Letters, 1, 7

McCarthy, I. G., Babul, A., Bower, R. G., \& Balogh, M. L. 2008, MNRAS, 386, 1309

McCarthy, I. G., Bower, R. G., Balogh, M. L., et al. 2007, MNRAS, 376, 497

McCarthy, I. G., Schaye, J., Ponman, T. J., et al. 2010, MNRAS, 406, 822

McKenzie, J. F. \& Voelk, H. J. 1982, A\&A, 116, 191

McNamara, B. R. \& Nulsen, P. E. J. 2007, ARAA, 45, 117

Miniati, F., Ryu, D., Kang, H., et al. 2000, ApJ, 542, 608

Mo, H., van den Bosch, F. C., \& White, S. 2010, Galaxy Formation and Evolution

Mortlock, D. J., Warren, S. J., Venemans, B. P., et al. 2011, Nature, 474, 616

Muanwong, O., Thomas, P. A., Kay, S. T., Pearce, F. R., \& Couchman, H. M. P. 2001, ApJ, 552, L27

Murray, S. G., Power, C., \& Robotham, A. S. G. 2013, MNRAS, 434, L61

Nagai, D. \& Kravtsov, A. V. 2004, in IAU Colloq. 195: Outskirts of Galaxy Clusters: Intense Life in the Suburbs, ed. A. Diaferio, 296-298

Nagai, D., Kravtsov, A. V., \& Kosowsky, A. 2003, ApJ, 587, 524

Nagai, D., Kravtsov, A. V., \& Vikhlinin, A. 2007a, ApJ, 668, 1

Nagai, D., Vikhlinin, A., \& Kravtsov, A. V. 2007b, ApJ, 655, 98

Navarro, J. F., Frenk, C. S., \& White, S. D. M. 1995, MNRAS, 275, 720

Norman, M. L. \& Bryan, G. L. 1999, in Lecture Notes in Physics, Berlin Springer Verlag, Vol. 530, The Radio Galaxy Messier 87, ed. H.-J. Röser \& K. Meisenheimer, 106

O’Hara, T. B., Mohr, J. J., Bialek, J. J., \& Evrard, A. E. 2006, ApJ, 639, 64

Omma, H., Binney, J., Bryan, G., \& Slyz, A. 2004, MNRAS, 348, 1105

Omukai, K. 2001, ApJ, 546, 635

Omukai, K. \& Inutsuka, S.-i. 2002, MNRAS, 332, 59

Omukai, K., Tsuribe, T., Schneider, R., \& Ferrara, A. 2005, ApJ, 626, 627

Onions, J., Knebe, A., Pearce, F. R., et al. 2012, MNRAS, 423, 1200

O'Shea, B. W., Bryan, G., Bordner, J., et al. 2004, ArXiv Astrophysics e-prints

Osmond, J. P. F. \& Ponman, T. J. 2004, MNRAS, 350, 1511

Palla, F., Salpeter, E. E., \& Stahler, S. W. 1983, ApJ, 271, 632

Pareschi, G. \& Campana, S. 2011, Memorie della Societa Astronomica Italiana Supplementi, 17, 16

Pearce, F. R., Thomas, P. A., Couchman, H. M. P., \& Edge, A. C. 2000, MNRAS, 317, 1029

Peebles, P. J. E. 1968, ApJ, 153, 1

Peebles, P. J. E. 1993, Principles of Physical Cosmology

Peterson, J. R. \& Fabian, A. C. 2006, Phys. Rep., 427, 1

Peterson, J. R., Kahn, S. M., Paerels, F. B. S., et al. 2003, ApJ, 590, 207

Pfrommer, C., Enßlin, T. A., \& Springel, V. 2008, MNRAS, 385, 1211

Pfrommer, C., Enßlin, T. A., Springel, V., Jubelgas, M., \& Dolag, K. 2007, MNRAS, 378, 385

Pfrommer, C., Springel, V., Enßlin, T. A., \& Jubelgas, M. 2006, MNRAS, 367, 113

Piffaretti, R., Jetzer, P., Kaastra, J. S., \& Tamura, T. 2005, A\&A, 433, 101

Pillepich, A., Porciani, C., \& Reiprich, T. H. 2012, MNRAS, 422, 44

Planck Collaboration, Ade, P. A. R., Aghanim, N., et al. 2013a, ArXiv e-prints

Planck Collaboration, Ade, P. A. R., Aghanim, N., et al. 2013b, ArXiv e-prints

Planelles, S., Borgani, S., Dolag, K., et al. 2013a, MNRAS, 431, 1487

Planelles, S., Borgani, S., Fabjan, D., et al. 2013b, MNRAS 
Planelles, S. \& Quilis, V. 2013, MNRAS, 428, 1643

Ponman, T. J., Bourner, P. D. J., Ebeling, H., \& Böhringer, H. 1996, MNRAS, 283, 690

Ponman, T. J., Sanderson, A. J. R., \& Finoguenov, A. 2003, MNRAS, 343, 331

Pratt, G. W., Arnaud, M., Piffaretti, R., et al. 2010, A\&A, 511, A85

Pratt, G. W., Böhringer, H., Croston, J. H., et al. 2007, A\&A, 461, 71

Pratt, G. W., Croston, J. H., Arnaud, M., \& Böhringer, H. 2009, A\&A, 498, 361

Press, W. H. \& Schechter, P. 1974, ApJ, 187, 425

Prieto, J., Jimenez, R., \& Haiman, Z. 2013, MNRAS, 436, 2301

Puchwein, E., Sijacki, D., \& Springel, V. 2008, ApJ, 687, L53

Puchwein, E., Springel, V., Sijacki, D., \& Dolag, K. 2010, MNRAS, 406, 936

Pudritz, R. E. \& Silk, J. 1989, ApJ, 342, 650

Puy, D. \& Signore, M. 2007, New Astronomy Reviews, 51, 411

Quilis, V. 2004, MNRAS, 352, 1426

Quilis, V., Bower, R. G., \& Balogh, M. L. 2001, MNRAS, 328, 1091

Quilis, V., Ibanez, J. M. A., \& Saez, D. 1998, ApJ, 502, 518

Ragone-Figueroa, C., Granato, G. L., Murante, G., Borgani, S., \& Cui, W. 2013, MNRAS, 436, 1750

Reed, D. S., Bower, R., Frenk, C. S., Jenkins, A., \& Theuns, T. 2007, MNRAS, 374, 2

Rees, M. J. 1984, ARAA, 22, 471

Regan, J. A. \& Haehnelt, M. G. 2009a, MNRAS, 396, 343

Regan, J. A. \& Haehnelt, M. G. 2009b, MNRAS, 393, 858

Regan, J. A., Johansson, P. H., \& Haehnelt, M. G. 2014, MNRAS, 439, 1160

Reiprich, T. H. \& Böhringer, H. 2002, ApJ, 567, 716

Ricker, P. M. \& Sarazin, C. L. 2001, ApJ, 561, 621

Roettiger, K., Stone, J. M., \& Burns, J. O. 1999, ApJ, 518, 594

Rosner, R. \& Tucker, W. H. 1989, ApJ, 338, 761

Rozo, E., Wechsler, R. H., Rykoff, E. S., et al. 2010, ApJ, 708, 645

Rudd, D. H., Zentner, A. R., \& Kravtsov, A. V. 2008, ApJ, 672, 19

Ryu, D., Kang, H., Hallman, E., \& Jones, T. W. 2003, ApJ, 593, 599

Safranek-Shrader, C., Bromm, V., \& Milosavljević, M. 2010, ApJ, 723, 1568

Sanderson, A. J. R., O’Sullivan, E., \& Ponman, T. J. 2009, MNRAS, 395, 764

Sanderson, A. J. R., Ponman, T. J., Finoguenov, A., Lloyd-Davies, E. J., \& Markevitch, M. 2003, MNRAS, 340, 989

Sanderson, A. J. R., Ponman, T. J., \& O’Sullivan, E. 2006, MNRAS, 372, 1496

Sarazin, C. L. 1986, Reviews of Modern Physics, 58, 1

Sarazin, C. L. 1988, Sky and Telescope, 76, 639

Sarazin, C. L. 2008, in Lecture Notes in Physics, Berlin Springer Verlag, Vol. 740, A Pan-

Chromatic View of Clusters of Galaxies and the Large-Scale Structure, ed. M. Plionis,

O. López-Cruz, \& D. Hughes, 1-4020

Saslaw, W. C. \& Zipoy, D. 1967, Nature, 216, 976

Schleicher, D. R. G., Banerjee, R., Sur, S., et al. 2010a, A\&A, 522, A115

Schleicher, D. R. G., Galli, D., Palla, F., et al. 2008, A\&A, 490, 521

Schleicher, D. R. G., Palla, F., Ferrara, A., Galli, D., \& Latif, M. 2013, A\&A, 558, A59

Schleicher, D. R. G., Spaans, M., \& Glover, S. C. O. 2010b, ApJ, 712, L69

Schmidt, W., Niemeyer, J. C., \& Hillebrandt, W. 2006, A\&A, 450, 265

Schneider, R., Ferrara, A., \& Salvaterra, R. 2004, MNRAS, 351, 1379

Schneider, R., Omukai, K., Limongi, M., et al. 2012, MNRAS, 423, L60

Schober, J., Schleicher, D., Federrath, C., et al. 2012, ApJ, 754, 99

Schure, K. M., Bell, A. R., O'C Drury, L., \& Bykov, A. M. 2012, Sp. Sc. Rev., 173, 491 
Shang, C., Bryan, G. L., \& Haiman, Z. 2010, MNRAS, 402, 1249

Shapiro, S. L. \& Teukolsky, S. A. 1986, Black Holes, White Dwarfs and Neutron Stars: The Physics of Compact Objects

Sharma, P., McCourt, M., Quataert, E., \& Parrish, I. J. 2012, MNRAS, 420, 3174

Sheth, R. K., Mo, H. J., \& Tormen, G. 2001, MNRAS, 323, 1

Sheth, R. K. \& Tormen, G. 1999, MNRAS, 308, 119

Short, C. J., Thomas, P. A., Young, O. E., et al. 2010, MNRAS, 408, 2213

Sijacki, D., Pfrommer, C., Springel, V., \& Enßlin, T. A. 2008, MNRAS, 387, 1403

Sijacki, D., Springel, V., Di Matteo, T., \& Hernquist, L. 2007, MNRAS, 380, 877

Silk, J. 2013, ApJ, 772, 112

Silk, J. \& Langer, M. 2006, MNRAS, 371, 444

Silk, J. \& Mamon, G. A. 2012, Research in Astronomy and Astrophysics, 12, 917

Simionescu, A., Allen, S. W., Mantz, A., et al. 2011, Science, 331, 1576

Skillman, S. W., O'Shea, B. W., Hallman, E. J., Burns, J. O., \& Norman, M. L. 2008, ApJ, 689,1063

Somerville, R. S., Gilmore, R. C., Primack, J. R., \& Domínguez, A. 2012, MNRAS, 423, 1992

Somerville, R. S., Hopkins, P. F., Cox, T. J., Robertson, B. E., \& Hernquist, L. 2008, MNRAS, 391, 481

Spaans, M. \& Silk, J. 2006, ApJ, 652, 902

Springel, V. 2005, MNRAS, 364, 1105

Springel, V., Di Matteo, T., \& Hernquist, L. 2005, MNRAS, 361, 776

Springel, V. \& Hernquist, L. 2003, MNRAS, 339, 289

Stacy, A., Greif, T. H., \& Bromm, V. 2010, MNRAS, 403, 45

Stancil, P. C., Lepp, S., \& Dalgarno, A. 1998, ApJ, 509, 1

Stanek, R., Rudd, D., \& Evrard, A. E. 2009, MNRAS, 394, L11

Sun, M., Voit, G. M., Donahue, M., et al. 2009, ApJ, 693, 1142

Sunyaev, R. A. \& Zeldovich, Y. B. 1972, Comments on Astrophysics and Space Physics, 4, 173

Sur, S., Federrath, C., Schleicher, D. R. G., Banerjee, R., \& Klessen, R. S. 2012, MNRAS, 423, 3148

Sur, S., Schleicher, D. R. G., Banerjee, R., Federrath, C., \& Klessen, R. S. 2010, ApJ, 721, L134

Susa, H. 2013, ApJ, 773, 185

Tan, J. C. \& Blackman, E. G. 2004, ApJ, 603, 401

Tasker, E. J. \& Tan, J. C. 2009, ApJ, 700, 358

Tegmark, M., Silk, J., Rees, M. J., et al. 1997, ApJ, 474, 1

The Fermi-LAT Collaboration, Ackermann, M., Ajello, M., et al. 2013, ArXiv e-prints

Tinker, J., Kravtsov, A. V., Klypin, A., et al. 2008, ApJ, 688, 709

Tinker, J. L., Robertson, B. E., Kravtsov, A. V., et al. 2010, ApJ, 724, 878

Tornatore, L., Borgani, S., Dolag, K., \& Matteucci, F. 2007, MNRAS, 382, 1050

Tozzi, P. \& Norman, C. 2001, ApJ, 546, 63

Treumann, R. A. 2009, Astron. Astrophys. Reviews, 17, 409

Truelove, J. K., Klein, R. I., McKee, C. F., et al. 1997, ApJL, 489, L179

Turk, M. J., Clark, P., Glover, S. C. O., et al. 2011, ApJ, 726, 55

Turk, M. J., Oishi, J. S., Abel, T., \& Bryan, G. L. 2012, ApJ, 745, 154

Valdarnini, R. 2002, ApJ, 567, 741

van Weeren, R. J., Röttgering, H. J. A., Bagchi, J., et al. 2009, A\&A, 506, 1083

Vazza, F., Brüggen, M., Gheller, C., \& Brunetti, G. 2012, MNRAS, 421, 3375 
Vazza, F., Brunetti, G., Gheller, C., Brunino, R., \& Brüggen, M. 2011, A\&A, 529, A17

Vazza, F., Brunetti, G., Kritsuk, A., et al. 2009, A\&A, 504, 33

Vikhlinin, A., Burenin, R. A., Ebeling, H., et al. 2009, ApJ, 692, 1033

Vikhlinin, A., Kravtsov, A., Forman, W., et al. 2006, ApJ, 640, 691

Vikhlinin, A., Markevitch, M., Murray, S. S., et al. 2005, ApJ, 628, 655

Vladimirov, A. E., Bykov, A. M., \& Ellison, D. C. 2008, ApJ, 688, 1084

Vladimirov, A. E., Bykov, A. M., \& Ellison, D. C. 2009, ApJ, 703, L29

Voit, G. M. 2005, Reviews of Modern Physics, 77, 207

Voit, G. M. 2011, ApJ, 740, 28

Voit, G. M., Balogh, M. L., Bower, R. G., Lacey, C. G., \& Bryan, G. L. 2003, ApJ, 593, 272

Voit, G. M. \& Bryan, G. L. 2001, Nature, 414, 425

Voit, G. M., Bryan, G. L., Balogh, M. L., \& Bower, R. G. 2002, ApJ, 576, 601

Volonteri, M. \& Bellovary, J. 2012, Reports on Progress in Physics, 75, 124901

Walker, S. A., Fabian, A. C., Sanders, J. S., \& George, M. R. 2012, MNRAS, 424, 1826

Wang, H.-H., Klessen, R. S., Dullemond, C. P., van den Bosch, F. C., \& Fuchs, B. 2010, MNRAS, 407, 705

Warren, M. S., Abazajian, K., Holz, D. E., \& Teodoro, L. 2006, ApJ, 646, 881

Watson, W. A., Iliev, I. T., D’Aloisio, A., et al. 2013, MNRAS, 433, 1230

Weinberg, D. H., Mortonson, M. J., Eisenstein, D. J., et al. 2013, Phys. Rep., 530, 87

White, M. 2002, ApJS, 143, 241

Winske, D., Thomas, V. A., Omidi, N., \& Quest, K. B. 1990, J. Geophys. Res., 95, 18821

Wise, J. H. \& Abel, T. 2008, ApJ, 685, 40

Wise, J. H., Turk, M. J., \& Abel, T. 2008, ApJ, 682, 745

Yang, H.-Y. K., Ricker, P. M., \& Sutter, P. M. 2009, ApJ, 699, 315

Yoshida, N., Omukai, K., \& Hernquist, L. 2008, Science, 321, 669

Yoshida, N., Omukai, K., Hernquist, L., \& Abel, T. 2006, ApJ, 652, 6

Zakamska, N. L. \& Narayan, R. 2003, ApJ, 582, 162 